\title{
LA INFLUENCIA DEL CONTEXTO EN LOS USOS DE LAS HERRAMIENTAS VIRTUALES
}

Resumen: Este artículo es producto de una investigación que ha perseguido los siguientes objetivos: Conocer los usos de las herramientas virtuales por una población representativa en edades, profesiones, género y formación. Identificar las dificultades que encuentran y buscar las causas que las originan .Valorar las ventajas de cada una de las herramientas en la vida profesional, en el ocio y en la labor formativa.

Se ha elegido un muestreo por cuotas, fundamentalmente, por el rigor que otorga.

Las herramientas de toma de datos elaboradas y aplicadas - protocolo de cuestionario, guión de entrevista y grupo de discusión- posibilitan traspasar la frontera del análisis cuantitativo versus cualitativo para adentrarse en una metodología mixta.

Un aprovechamiento acertado, rápido, seguro, selectivo de las herramientas virtuales estudiadas representa un principio de innovación, acceso al conocimiento y desarrollo de la actuación profesional. La cualificación en competencias básicas para construir, transferir y procesar conocimientos especializados y generales que circulan por la red, son de utilidad a lo largo de la vida.

Palabras clave. Herramientas virtuales, competencias, usos, ventajas, dificultades, formación e innovación

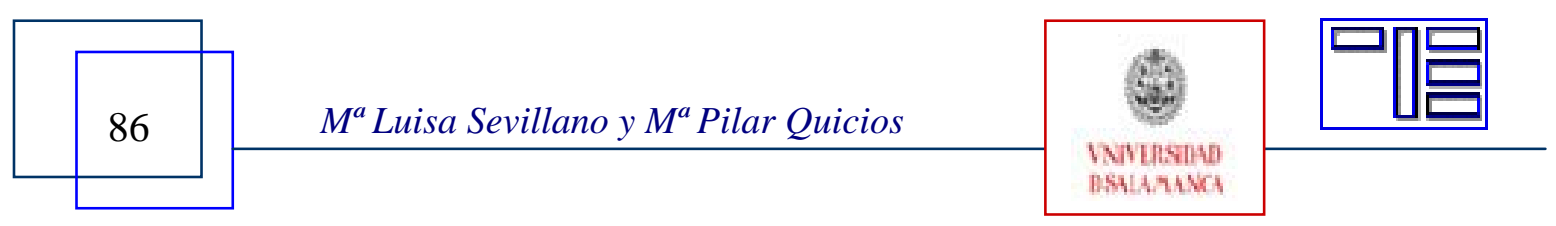




\section{THE INFLUENCE OF CONTEXT IN VIRTUAL TOOL USING.}

Abstract: This article is the product of an investigation that has pursued the following objectives: To know the uses of virtual tools by a representative population in age, profession, gender and formation. To identify the difficulties they encounter and to look for their causes. To assess the advantages of every tool in professional life, in leisure time and in teaching work.

A sampling by quota has been chosen, basically for its rigor.

The data acquisition tools -questionnaire protocol, interview script, and discussion group- than have been developed and applied make it possible to go beyond the frontier of quantitative vs qualitative analysis and to advance into a mixed methodology.

A quick, sure, selective, pertinent use of the studied virtual tools represents a principle of innovation, access to knowledge and development of professional performance.

The qualifying in basic competences for building, transferring and processing general and specialized knowledge that can be found on the net and that is useful throughout one's life.

Keywords: Virtual tools. Competences. Uses. Advantages. Difficulties. Professional training. Innovation.

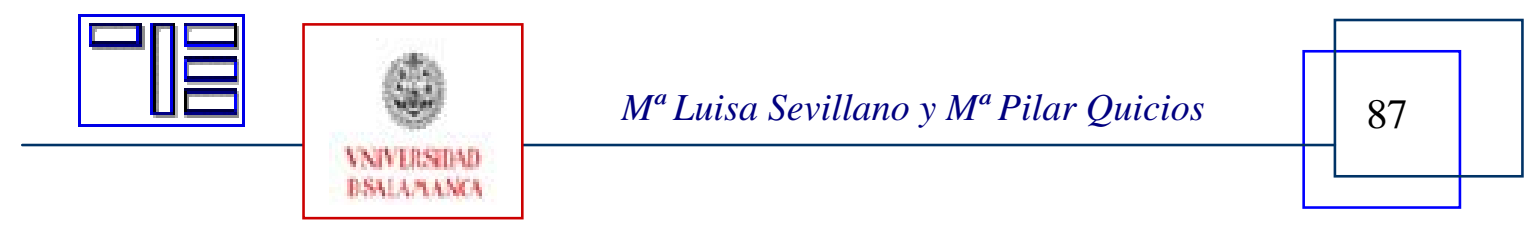




\section{LA INFLUENCIA DEL CONTEXTO EN LOS USOS DE LAS HERRAMIENTAS VIRTUALES}

Dra. María Luisa Sevillano García

mlsevillano@edu.uned.es

Dra. María del Pilar Quicios García

pquicios@edu.uned.es

Universidad Nacional de Educación a Distancia

\section{PLANTEAMIENTO DEL PROBLEMA}

El manejo acertado, rápido, seguro y selectivo de las herramientas virtuales constituye, hoy en día, tanto un principio de innovación como un modo diferente de acceso al conocimiento y al desarrollo de la actuación profesional a lo largo de la vida. Esta innegable realidad permite afirmar que el uso de las herramientas virtuales se ha convertido en un reto continuo e ineludible en la actual formación permanente cualificada. Igualmente, se puede comprender que el manejo de este tipo de herramientas se considere una competencia que es preciso cultivar y hasta potenciar en la dimensión vital del ocio, del entretenimiento y de la vida integrada en la comunidad ciudadana.

En estos momentos la sociedad demanda personas cualificadas en competencias básicas. Usuarios capaces de construir, transferir y procesar de forma continuada, tanto conocimientos especializados como generales que circulan por la red. A su vez se precisa el desarrollo de competencias a lo largo de la vida para utilizar inteligentemente el abanico de recursos disponibles.

Ya se pueden formular propuestas y marcos de intervención educativa basados en los datos procedentes de la investigación y sobre ellos capacitar a las personas en las habilidades que demandan los nuevos modos de relación e interacción con las tecnologías. Ofrecer a los agentes encargados de diseñar e implementar ese tipo de procesos formativos -instituciones, corporaciones locales, centros educativos, etcétera- un modelo que, contribuya a consensuar y estandarizar la práctica pedagógica en este campo.

Para ayudar a estos fines, llevamos a cabo un proyecto de investigación profesores de seis Universidades titulado Diagnóstico y desarrollo de competencias en el uso de herramientas de comunicación virtuales para la sociedad del conocimiento a lo largo

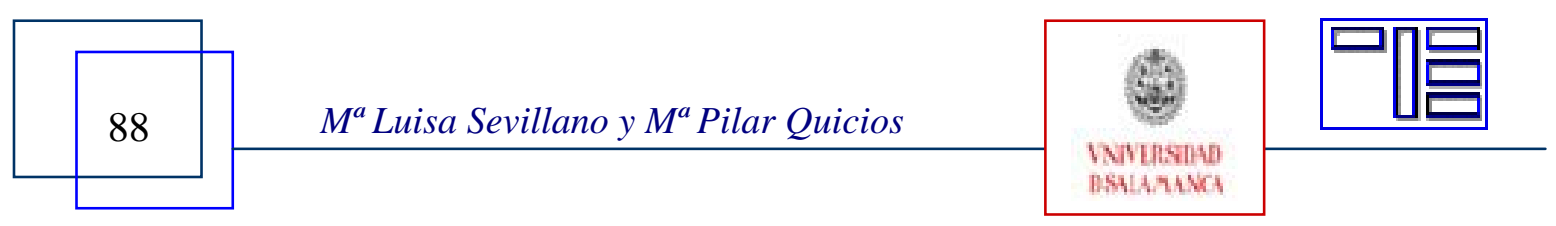


de la vida (N. de Referencia SEJ-2004-0680 durante cuatro años y financiado por el Ministerio de Ciencia e Innovación dentro del programa, Proyectos I+D, acciones estratégicas y errantes, del cual forma parte esta contribución

\section{ANTECEDENTES Y FUNDAMENTACIÓN TEÓRICA}

La discusión y preocupación sobre cuestiones análogas ya ha estado presente en los últimos tiempos en la comunidad científica internacional y nacional. A título de ejemplo enumeramos algunos casos: María Gerhard y Walter Klingler (2006) investigan y publican sobre Medienerziehung in der Zukunft, ( Formación medial en el futuro ) en la revista Media Perspektiven, concluyendo que Internet es valorado especialmente por la combinación de ofertas de contenidos y posibilidades que ofrece en cuanto a formas diversas de comunicación, así como su potencial de proveedor para otros medios. Gros y Mariella (2007) realizan una investigación que se centra en el uso de la plataforma utilizada por las profesoras en el proceso de incorporación de herramientas virtuales: "Interacción e influencia educativa: la construcción del conocimiento en entornos electrónicos de enseñanza y aprendizaje" es su título y se trata de Investigación subvencionada por la Comisión Interministerial de Ciencia y Tecnología (proyecto BSO20013680-CO2-0).

En el Proyecto La VccSSe-Virtual Community-Colllaborating Space for Science Education. http://vccsse.ssai.valahia.ro/main/index , participan como institución coordinadora la Universidad valahia de Targoviste ( Rumania ) y como socios el Centro de formación del profesorado de Zaragoza (España), Centro de formación permanente del profesorado "wom" en Bielsko-biala ( Polonia ) Universidad Babes-bolyai en Cluj Napoca ( Rumanía ), Universidad tecnológica de Varsovia ( Polonia) ,Universidad de Joensuu ( Finlandia) Universidad de Patras ( Grecia). Todos los investigadores implicados buscan los siguientes objetivos:

1. Ofrecer a los profesores en su formación permanente una técnica específica basada en Instrumentos Virtuales

2. Aplicar las metodologías de enseñanza desarrolladas a un Espacio de Cooperación Virtual

3. Desarrollar la cooperación y conciencia europea.

Estudio sobre el uso de los foros virtuales para favorecer las actividades colaborativas en la enseñanza superior.(Sevillano, M. L. (2006) Competencias y Conocimientos sobre Herramientas virtuales Formato de archivo: PDF/Adobe Acrobat - Versión en HTML www.formatex.org/micte2006/pdf/27-31.pdf -.

Chan (2005) desde un paradigma comunicacional, se plantea el tipo de competencias requeridas para diseñar y actuar en un entorno digital. Como nuevo espacio social requiere constructores, actores y no sólo navegantes, observadores o lectores que consumen lo que otros producen. El desarrollo de nuevas competencias de los sujetos para

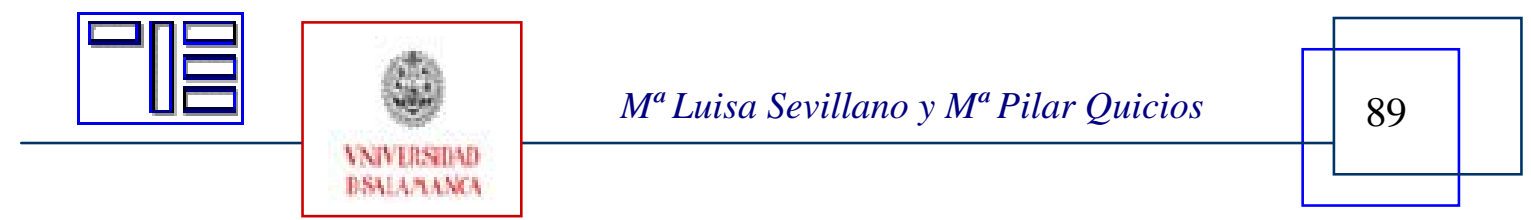


relacionarse con objetos de conocimiento en entornos digitales se percibe como parte de un fenómeno de transformación de las prácticas sociales, y entre ellas las educativas. (http://redie.uabc.mx/vol7no2/contenido-chan.html)

La Propuesta de Recomendación del Parlamento Europeo y del Consejo sobre las competencias clave para el aprendizaje permanente (COM, 2005), contempla la competencia digital para el trabajo, el ocio y la comunicación. Esta competencia se entiende como un conjunto de capacidades que nos deben llevar a una valoración crítica de las mismas aportaciones provenientes de la redes (Rüdiger 2005:87). Tulodzieki (1999:302) indica en esta misma línea que deberían reunir cinco aspectos:

1. Elección y uso de lo presentado en redes para el ocio, juego, aprendizaje, formación, solución de problemas, toma de decisiones, información política y alternativas de acción.

2. Elaboración y distribución de productos para ser distribuidos por la red.

3. Comprensión y valoración de los lenguajes virtuales, sus sistemas de signos, técnicas de elaboración y representación.

4. Reconocimiento de los influjos de las redes en el ámbito de los sentimientos, las representaciones falsas, orientaciones no adecuadas en el uso y campo de los valores y los comportamientos.

5. Enjuiciamiento de las condiciones técnicas, económicas, legales, sociales, institucionales y políticas en la producción y distribución de mensajes para y en la red.

Para Liegle y Meso (2001) las herramientas virtuales son aquellas aplicaciones utilizadas para la distribución de conocimiento a un conjunto definido de personas vía WWW. Collis y Moonen (2007) las entienden y definen como un paquete de software integrado que ofrece algunos o la totalidad de aspectos de preparación, distribución e interacción de información y permite que estos aspectos sean accesibles vía redes. La comunicación virtual mediante herramientas virtuales es el intercambio de información en entornos virtuales.

En este estudio presentaremos diferentes formas de comunicarse virtualmente como mensajería instantánea, correo electrónico, videoconferencia, blogs, chats, foros, otros. Estos núcleos de intercambio de comunicación tienen en común que nos permiten interactuar con personas de diversos puntos del planeta aunque físicamente no estén presentes.

\section{DISEÑO Y METODOLOGÍA DE LA INVESTIGACIÓN}

Un elemento primordial fue la selección de las unidades de observación, actuación de la que depende básicamente la calidad de la información que se recoja. Definida la población, se procedió al diseño de la muestra. El sistema de muestreo por cuotas fue el elegido por su condición de constituir una variedad de muestreo no probabilístico que par-

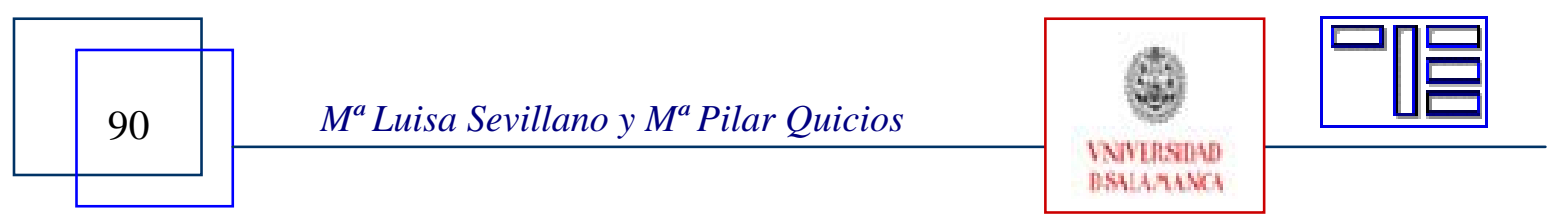


te de la segmentación de la población de interés en grupos, a partir de variables sociodemográficas relacionadas con los objetivos de la investigación. La opción se fundamentó también en el rigor que ofrece este tipo de muestreo. Determinada la metodología a seguir, la descripción de la muestra diferenciada por género y formación , quedó establecida como indican las tablas 1 y 2 :

\begin{tabular}{|l|l|l|l|l|}
\hline Edades & Estudios Obligatorios & Estudios Medios & Estudios Superiores & Totales \\
\hline Hasta 30 & 1 & 2 & 1 & 4 \\
\hline $30-45$ & 2 & 5 & 1 & 8 \\
\hline $46-65$ & 1 & 6 & 2 & 9 \\
\hline 66 y más & - & 1 & - & 1 \\
\hline Totales & 4 & 14 & 4 & 22 \\
\hline
\end{tabular}

Tabla 1. Muestreo hombres

\begin{tabular}{|l|l|l|l|l|}
\hline Edades & Estudios Obligatorios & Estudios Medios & Estudios Superiores & Totales \\
\hline Hasta 30 & - & 2 & 1 & 3 \\
\hline $30-45$ & 2 & 6 & 1 & 9 \\
\hline $46-65$ & 2 & 5 & 2 & 9 \\
\hline 66 y más & 1 & 1 & - & 2 \\
\hline Totales & 5 & 4 & 23 \\
\hline
\end{tabular}

La muestra final quedó conformada por 1311 personas: 602 hombres y 695 mujeres. Del resto, 14 personas no consta. La muestra elegida en la investigación resulta ser representativa en los diversos segmentos de edades, formación y ocupación según análisis estadísticos realizados.

El 45.1 \% de hombres y el 54.9 de mujeres se corresponden con la proporción de población a nivel de Estado. Por edades se han obtenido porcentajes correspondientes a los previstos en el muestreo aleatorio por cuotas: Hasta 25 años: 14.4 \%. De 25-35 años: 18.4 \%. De 35- 45 años: 26.7 \%. De 45-55 años: 20.7 \%. Más de 55 años: 19.1 \%

La distribución de la muestra por nivel de formación se corresponde, también, con lo previsto en los cálculos de representatividad por cuotas. Al grupo de estudios medios le corresponde el $52.2 \%$ de la muestra, seguidos de aquellos que tienen estudios superiores con el 24.6 \% del total. El grupo de estudios básicos está representado con el $20.1 \%$ de los encuestados siendo poco significativos los segmentos sin estudios con un $0.8 \%$ y cursando estudios $0.9 \%$ y otros estudios $3.4 \%$. Es preciso hacer constar que estos son los porcentajes que corresponden a cada estrato formativo en función de la población estudiada. Como se observa, el grado de formación de la muestra resulta ser alto.

Con respecto a la situación profesional anotemos que un 33.4 \% figura como estudiantes, seguido de un $22.2 \%$ que realiza labores del hogar. Un $17.0 \%$ son jubilados de más de 65 años, un $11.0 \%$ trabajadores, $8.4 \%$ pensionistas de menos de 65 años y un $7.9 \%$

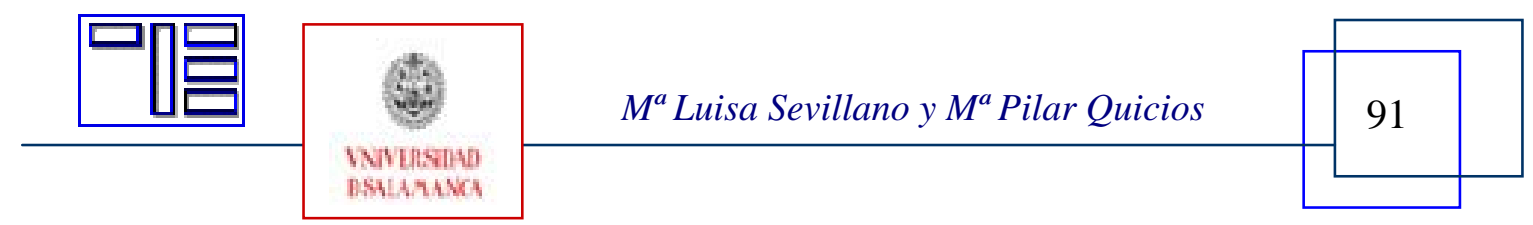


Revista Electrónica Teoría de la Educación.

Educación y Cultura en la Sociedad de la Información.

http://www.usal.es/teoriaeducacion

desempleados. Acerca de los conocimientos que tienen en informática un $70.2 \%$ estima que poseen conocimientos básicos, el $20.7 \%$ ninguno y el $9.2 \%$ elevados. En cuanto a formación en Tecnologías de la Información y la Comunicación 55.5 \% están formados.

Las herramientas de toma de datos elaboradas (protocolo del cuestionario, guión de entrevista y del grupo de discusión) posibilitan la utilización de una metodología mixta (cuantitativo-cualitativa). El manejo de más de un instrumento en la recogida de datos refuerza la solidez de los resultados y conclusiones obtenidas. Dadas las características del cuestionario, básicamente de opinión sobre ítems disjuntos sin pretensión de formar escalas, el estudio de fiabilidad se orienta en los aspectos de estabilidad. Para conseguir el objetivo anterior se procedió a seleccionar dos muestras aleatorias del colectivo encuestado. Se realizó un estudio frecuencista de las preguntas de opinión y un análisis de la varianza en las preguntas con respuesta de escala. Los resultados se muestran a continuación.

\begin{tabular}{|l|l|l|}
\hline Tecnologías y aparatos a los que accede en la vida cotidiana & ji-cuadrado & significación \\
\hline Acceso tecnologías_ordenador & 0,075 & 0,784 \\
\hline Acceso tecnologías_Internet & 0,106 & 0,745 \\
\hline Acceso tecnologías_TV analógica & 0,066 & 0,797 \\
\hline Acceso tecnologías_TV digital & 1,092 & 0,296 \\
\hline Acceso tecnologías_teléfono fijo & 0,165 & 0,684 \\
\hline Acceso tecnologías_teléfono móvil & 0,815 & 0,367 \\
\hline Acceso tecnologías_vídeo VHS & 0,183 & 0,669 \\
\hline Acceso tecnologías_DVD & 0,732 & 0,392 \\
\hline Acceso tecnologías_foto digital & 0,616 & 0,433 \\
\hline Acceso tecnologías_videocámara digital & 0,682 & 0,409 \\
\hline Acceso tecnologías_videoconsola & 1,000 & 0,317 \\
\hline Acceso tecnologías_PDA & 1,788 & 0,181 \\
\hline Acceso tecnologías_GPS & 0,268 & 0,605 \\
\hline
\end{tabular}

Tabla 3. Estudio de fiabilidad

Las poblaciones estudiadas de forma experimental (Jóvenes universitarios 18-25 años) manifiestan claras demandas de formación sistemática en todas y cada una de las herramientas virtuales. Más aún, se aprecia que tal formación no ha tenido lugar y que los conocimientos, destrezas y capacidades de los entrevistados responden principalmente a un autoaprendizaje, confiando a veces en que el uso y manejo traerá la formación.

\section{4.- OBJETIVOS}

1. Conocer usos frecuentes de diversas herramientas virtuales por parte de una población representativa en edades, profesiones, género, formación y ámbito territorial

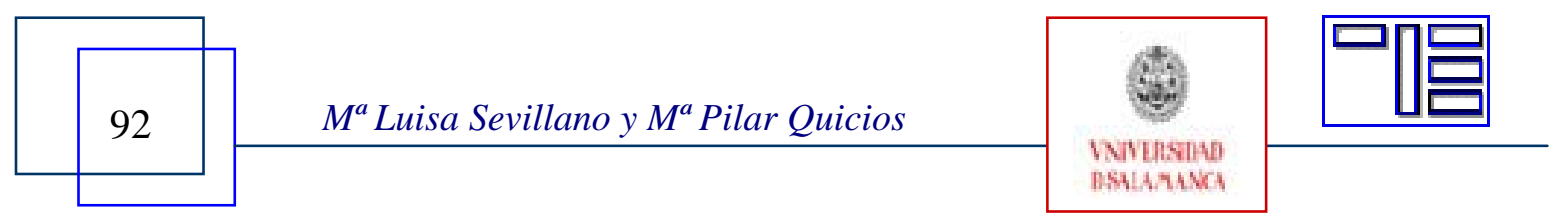


2. Identificar la influencia del contexto en los usos de cada una de las herramientas estudiadas

3. Identificar dificultades que encuentran en su utilización así como las causas que las originan

4. Valorar las ventajas verificadas en cada una de las herramientas

5. Determinar índices de aprovechamiento por parte de los diferentes segmentos de población estudiados de cada una de las herramientas virtuales objeto de análisis

\section{5.- RESULTADOS}

\section{1.- Dificultades que representan diferentes herramientas virtuales}

Las herramientas tenidas en consideración han sido: e-mail, foros, chat, Messenger, videoconferencia, blog, voz IP, teléfono móvil, sms. Para la obtención de los resultados, se pregunta a los encuestados, que han respondido que utilizan todas las herramientas, por la valoración que les merece las dificultades siguientes: velocidad en la comunicación, interactividad, conectividad/acceso, manejabilidad, eficacia, coste del servicio, opciones de movilidad y seguridad. Los resultados por tipología de dificultad se muestran a continuación.

Los blog y la voz IP son los medios donde los encuestados piensan que la velocidad de comunicación es el mayor inconveniente. (Tabla 4). De nuevo son los blog y la voz IP los medios donde los encuestados piensan que la interactividad es el mayor inconveniente. (Tabla 5). En esta ocasión es la videoconferencia y la voz IP los medios donde los encuestados piensan que el acceso es el mayor inconveniente. (Tabla 6). Repite la videoconferencia, los blog y la voz IP como medios donde los encuestados piensan que el manejo es el mayor inconveniente.(Tabla 7). Nuevamente blog y la voz IP son herramientas donde los encuestados piensan que la eficacia en el manejo es el mayor inconveniente. (Tabla 8). En esta ocasión es el teléfono móvil tanto para voz como para mensajes el medio donde los encuestados piensan que el coste del servicio es el mayor inconveniente. (Tabla 9). Para los encuestados es la videoconferencia y la voz IP donde las opciones de movilidad son el mayor inconveniente. (Tabla 10) y es el Chat el contexto de comunicación donde los encuestados piensan que la seguridad es el mayor inconveniente (Tabla 11)

\begin{tabular}{|l|l|l|l|l|l|l|l|l|l|}
\hline & E-mail & Foros & Chat & Messenger & $\begin{array}{l}\text { Videoconfe- } \\
\text { rencia }\end{array}$ & Blog & Voz IP & $\begin{array}{l}\text { Móvi } \\
\text { l }\end{array}$ & SMS \\
\hline $\bar{X}$ & 3,79 & 4,25 & 4,12 & 3,80 & 4,63 & 4,75 & 4,71 & 2,71 & 3,17 \\
\hline$\sigma$ & 3,274 & 3,407 & 3,607 & 3,546 & 3,600 & 3,586 & 3,687 & $\begin{array}{l}2,95 \\
2\end{array}$ & 2,992 \\
\hline
\end{tabular}

Tabla 4 Valoración de la dificultad encontrada en la velocidad en la Comunicación

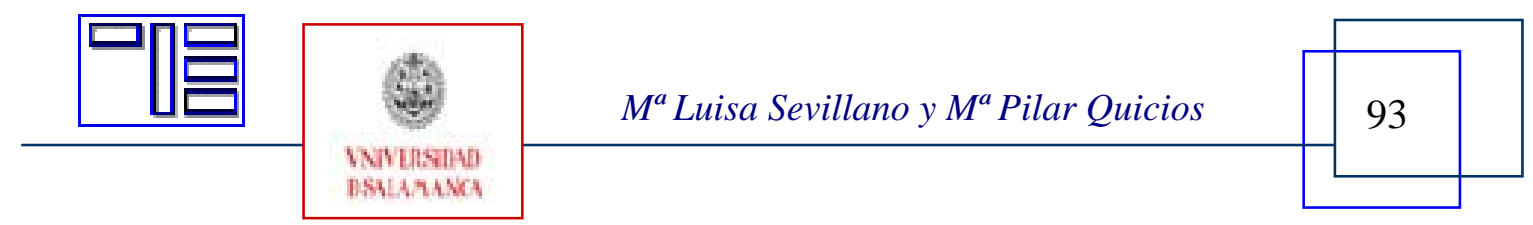


Revista Electrónica Teoría de la Educación.

Educación y Cultura en la Sociedad de la Información.

http://www.usal.es/teoriaeducacion

Vol.10. N². Julio 2009

\begin{tabular}{|l|l|l|l|l|l|l|l|l|l|}
\hline & E-mail & Foros & Chat & Messenger & Videoconferencia & Blog & $\begin{array}{l}\text { Voz } \\
\text { IP }\end{array}$ & Móvil & SMS \\
\hline $\bar{X}$ & 3,92 & 4,21 & 4,01 & 3,66 & 4,25 & 4,66 & 4,46 & 2,79 & 3,37 \\
\hline$\sigma$ & 3,145 & 3,372 & 3,509 & 3,498 & 3,598 & 3,503 & 3,622 & 2,920 & 2,972 \\
\hline
\end{tabular}

Tabla 5 Valoración de la dificultad encontrada en la interactividad

\begin{tabular}{|l|l|l|l|l|l|l|l|l|l|}
\hline & E-mail & Foros & Chat & Messenger & Videoconferencia & Blog & $\begin{array}{l}\text { Voz } \\
\text { IP }\end{array}$ & Móvil & SMS \\
\hline $\bar{X}$ & 3,78 & 4,13 & 4,17 & 3,90 & 4,88 & 4,51 & 4,74 & 2,96 & 3,04 \\
\hline$\sigma$ & 3,145 & 3,370 & 3,416 & 3,467 & 3,495 & 3,548 & 3,554 & 2,830 & 2,940 \\
\hline
\end{tabular}

Tabla 6 Valoración de la dificultad encontrada en la conectividad/acceso

\begin{tabular}{|l|l|l|l|l|l|l|l|l|l|}
\hline & E-mail & Foros & Chat & Messenger & Videoconferencia & Blog & $\begin{array}{l}\text { Voz } \\
\text { IP }\end{array}$ & Móvil & SMS \\
\hline $\bar{X}$ & 3,57 & 4,15 & 4,02 & 3,83 & 4,68 & 4,71 & 4,69 & 2,78 & 3,23 \\
\hline$\sigma$ & 3,224 & 3,400 & 3,461 & 3,506 & 3,509 & 3,611 & 3,610 & 2,872 & 3,024 \\
\hline
\end{tabular}

Tabla 7 Valoración de la dificultad encontrada en el manejo

\begin{tabular}{|l|l|l|l|l|l|l|l|l|l|}
\hline & E-mail & Foros & Chat & Messenger & Videoconferencia & Blog & $\begin{array}{l}\text { Voz } \\
\text { IP }\end{array}$ & Móvil & SMS \\
\hline $\bar{X}$ & 3,51 & 4,17 & 4,13 & 3,68 & 4,26 & 4,46 & 4,42 & 2,83 & 3,08 \\
\hline$\sigma$ & 3,211 & 3,423 & 3,459 & 3,440 & 3,506 & 3,549 & 3,624 & 2,950 & 2,934 \\
\hline
\end{tabular}

Tabla 8 Valoración de la dificultad encontrada en la eficacia

\begin{tabular}{|l|l|l|l|l|l|l|l|l|l|}
\hline & E-mail & Foros & Chat & Messenger & Videoconferencia & Blog & $\begin{array}{l}\text { Voz } \\
\text { IP }\end{array}$ & Móvil & SMS \\
\hline $\bar{X}$ & 4,14 & 4,08 & 4,13 & 4,02 & 4,85 & 4,43 & 4,63 & 5,77 & 5,12 \\
\hline$\sigma$ & 3,427 & 3,519 & 3,528 & 3,539 & 3,618 & 3,670 & 3,652 & 3,213 & 3,232 \\
\hline
\end{tabular}

Tabla 9 Valoración de la dificultad encontrada por coste del servicio

\begin{tabular}{|l|l|l|l|l|l|l|l|l|l|}
\hline & E-mail & Foros & Chat & Messenger & Videoconferencia & Blog & $\begin{array}{l}\text { Voz } \\
\text { IP }\end{array}$ & Móvil & SMS \\
\hline $\bar{X}$ & 4,38 & 4,51 & 4,53 & 4,39 & 4,97 & 4,70 & 4,91 & 2,74 & 2,88 \\
\hline$\sigma$ & 3,326 & 3,508 & 3,529 & 3,518 & 3,638 & 3,628 & 3,667 & 2,879 & 3,023 \\
\hline
\end{tabular}

Tabla 10 Valoración de la dificultad encontrada por opciones de movilidad

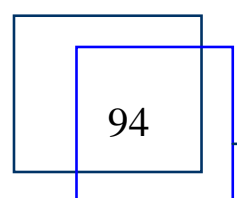

$M^{a}$ Luisa Sevillano y $M^{a}$ Pilar Quicios

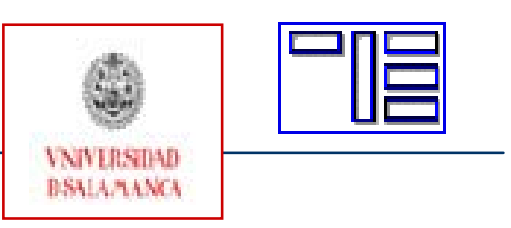




\begin{tabular}{|l|l|l|l|l|l|l|l|l|l|}
\hline & E-mail & Foros & Chat & Messenger & Videoconferencia & Blog & $\begin{array}{l}\text { Voz } \\
\text { IP }\end{array}$ & Móvil & SMS \\
\hline $\bar{X}$ & 4,76 & 4,84 & 5,35 & 4,81 & 4,77 & 4,85 & 4,84 & 3,24 & 3,40 \\
\hline$\sigma$ & 3,141 & 3,413 & 3,555 & 3,391 & 3,539 & 3,550 & 3,581 & 2,855 & 2,966 \\
\hline
\end{tabular}

Tabla 11 Valoración de la dificultad encontrada por seguridad

\section{2.- Utilización de diferentes herramientas virtuales en distintos contextos}

\subsection{1.- En el hogar}

Como puede observarse en el gráfico las tres herramientas mejor valoradas para su utilización en el hogar son: el teléfono móvil, el teléfono móvil para comunicarse por mensajes y el correo electrónico. Estas tres tienen una valoración de aprobado, aún cuando la última esté justo en el límite. La utilización de diversos medios en el entorno del hogar evidencia que dos (móvil y SMS ) han alcanzado un uso predominante, al obtener valores superiores a 5, con una ponderación cercana al 6 (5.99).

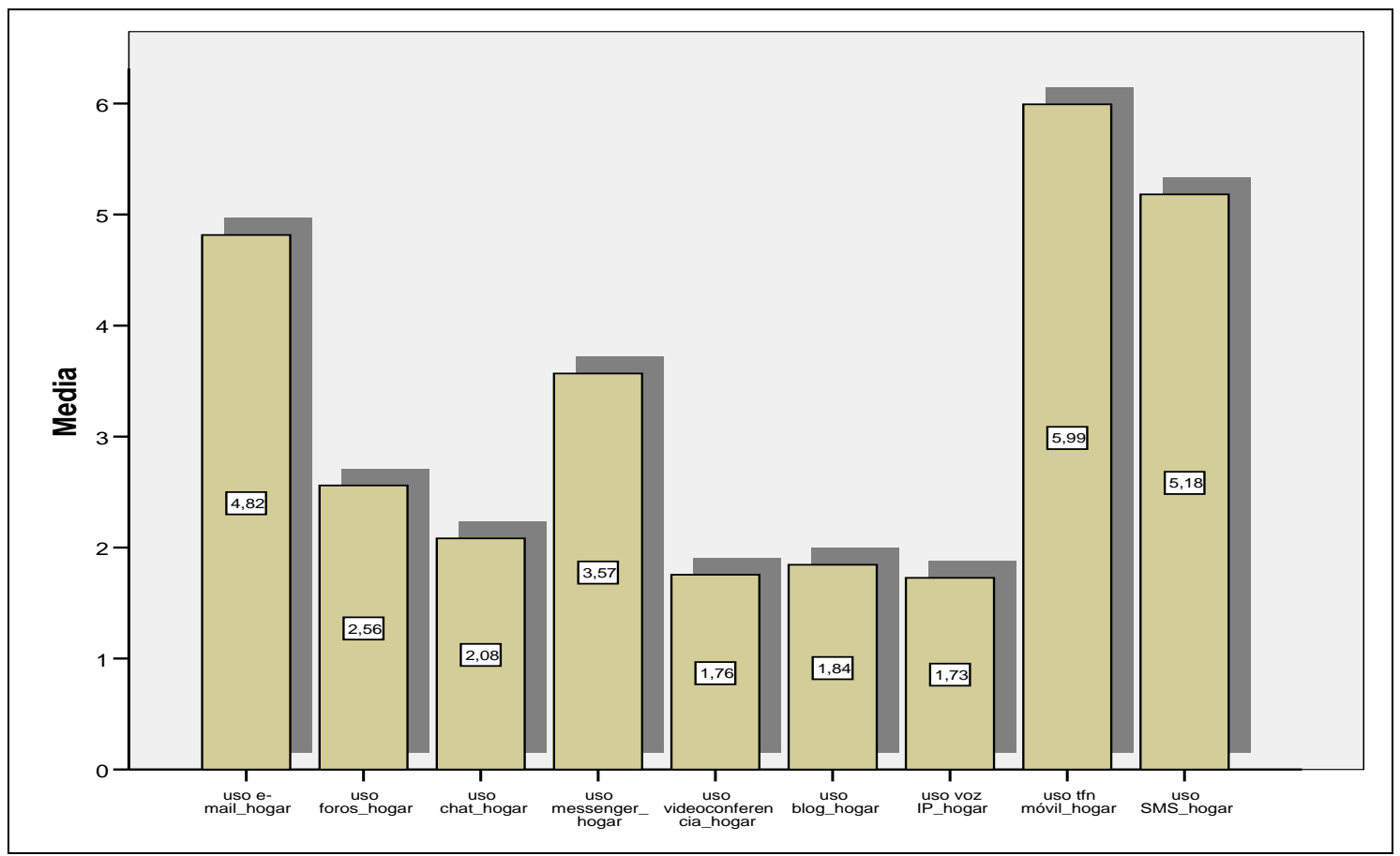

Gráfico 1 Hogar

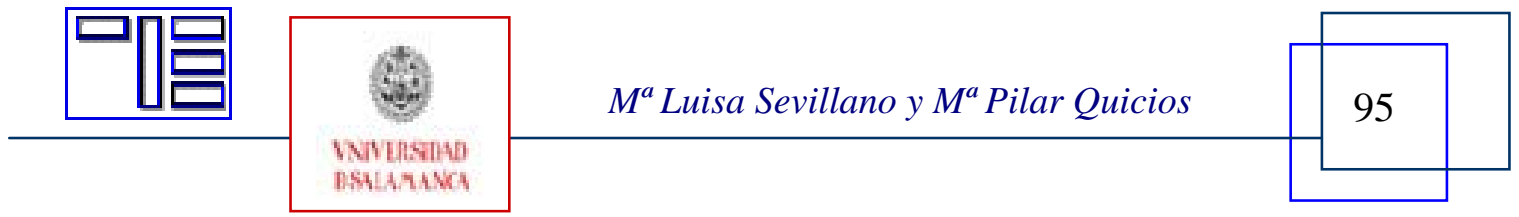


Revista Electrónica Teoría de la Educación.

Educación y Cultura en la Sociedad de la Información.

http://www.usal.es/teoriaeducacion

Vol.10. No2. Julio 2009

\subsection{2.- En el trabajo}

Un ámbito de claro nivel tecnológico ha de ser, sin lugar a dudas, el mundo laboral en el que el uso de la mayoría de estas herramientas ha de responder a las verdaderas demandas de un modelo en cambio globalizado.

En los escenarios laborales resaltan, nuevamente, el teléfono móvil y el SMS, aunque su uso se pospone ahora al del correo y emerge como el medio más apropiado aunque distante del teléfono móvil.

Los chat ocupan la valoración más lejana, seguida con una más elevada situación por el uso IP, videoconferencia y blog, Las puntuaciones globales son distantes de las alcanzadas en el contexto del hogar, en dos puntos.

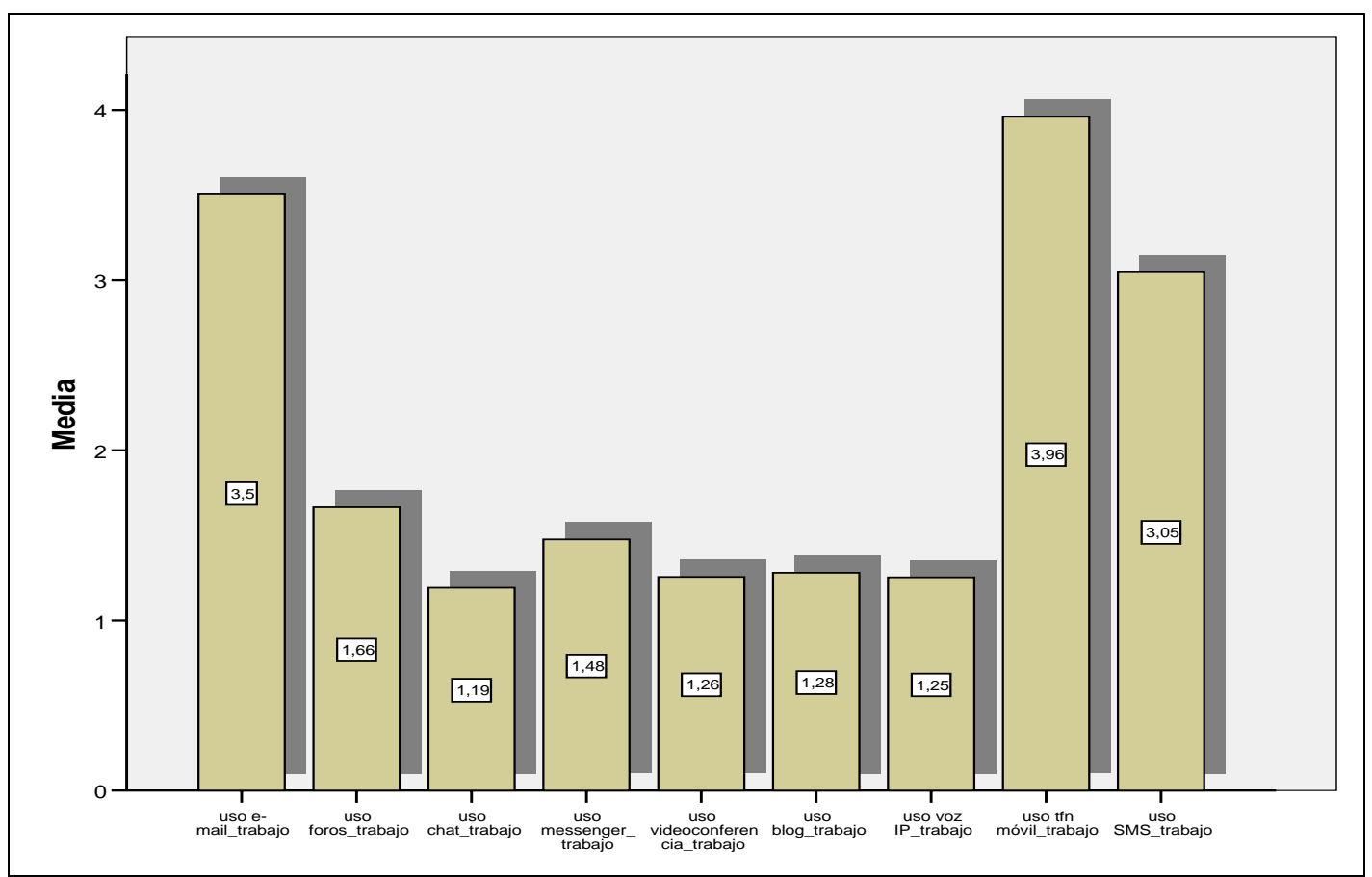

Gráfico 2 Trabajo

\subsection{3.- En la calle}

En este contexto sólo hay dos medios con valoración de aprobado: el teléfono móvil para hablar y dicho medio para mandar mensajes (SMS). La nueva generación de móviles está incorporando con gran potencialidad el uso de correos y de blog, así como de videoconferencia y Messenger.

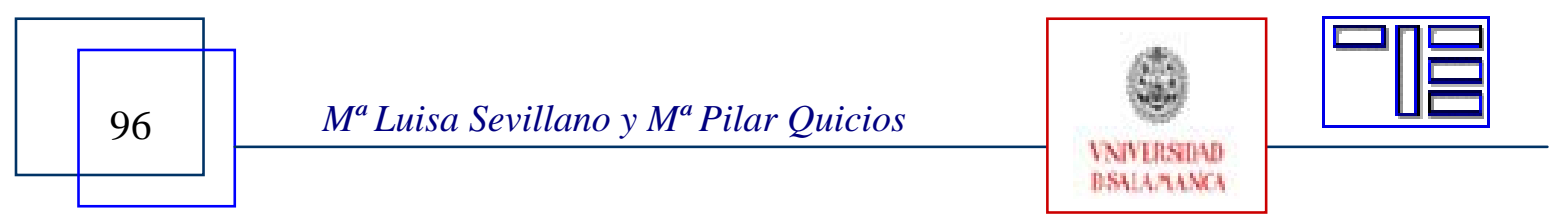




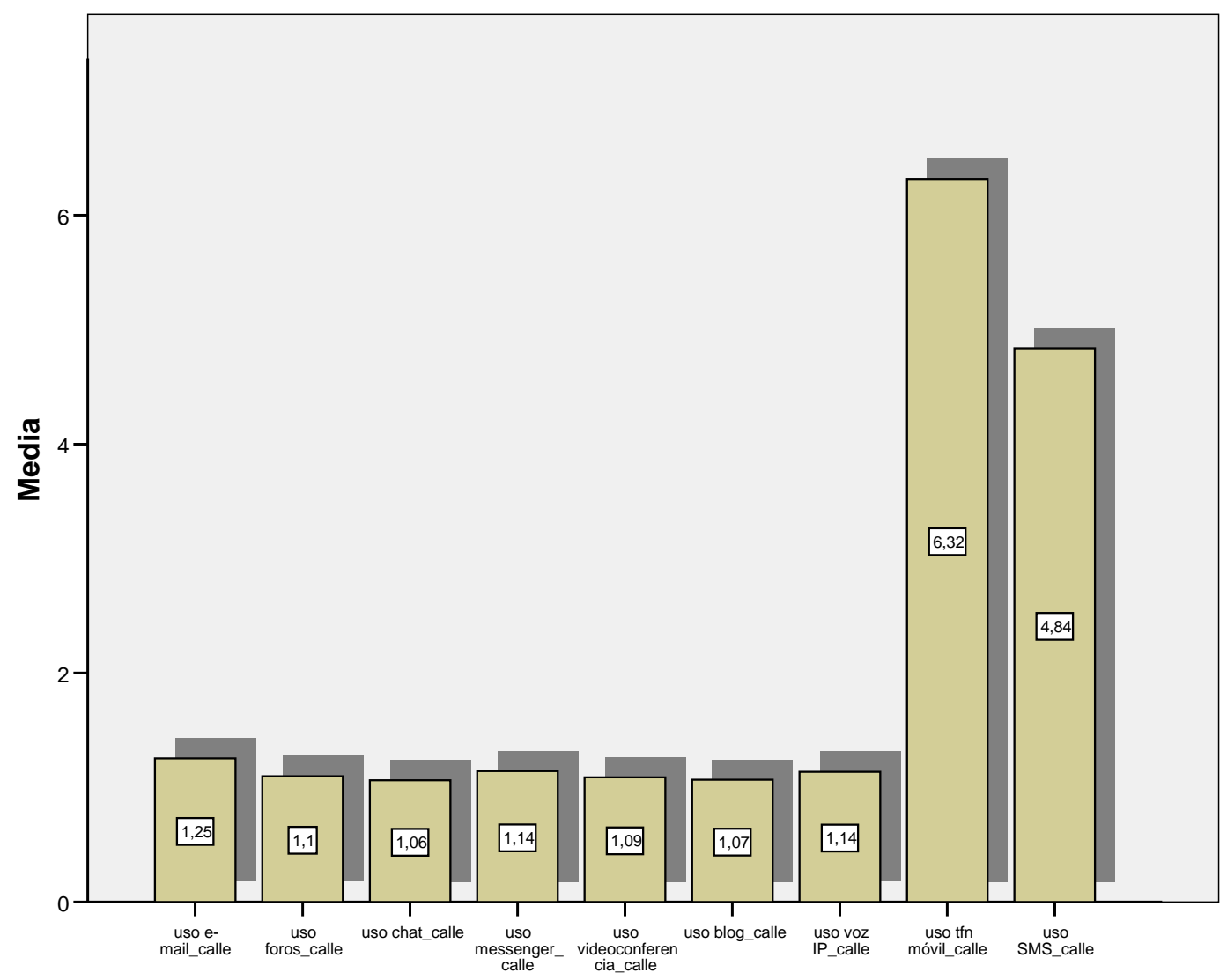

Gráfico 3 Calle

\subsection{4.- En la Escuela/ Instituto/ Facultad}

Siguen repitiéndose los tres medios mencionados en apartados anteriores, con una valoración, en este caso, más reducida. Se mantiene la tendencia al confirmar el uso del Messenger en el cuarto lugar y sigue en el último lugar de ponderación el uso de la voz IP y la videoconferencia.

La reflexión en los ambientes, hogar-familia y escolar-comunidad educativa, es que en ambos se mantiene el conjunto de valores, pero el uso del móvil y del Messenger, ha de reducirse a escenarios de recreo, salas de docentes, quizás esta realidad explique el descenso de uso. Sin embargo, en el centro educativo alcanza una similar valoración el uso del foro y del Messenger, que puedan significar la incorporación paulatina de estos medios a la institución formativa.

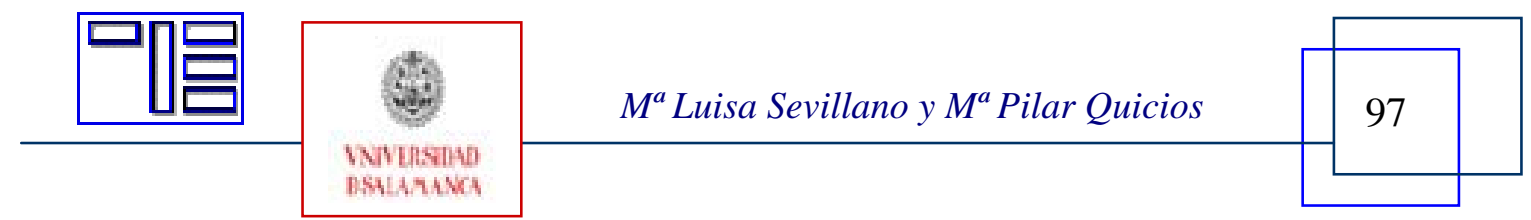


Revista Electrónica Teoría de la Educación.

Educación y Cultura en la Sociedad de la Información.

http://www.usal.es/teoriaeducacion

Vol.10. No2. Julio 2009

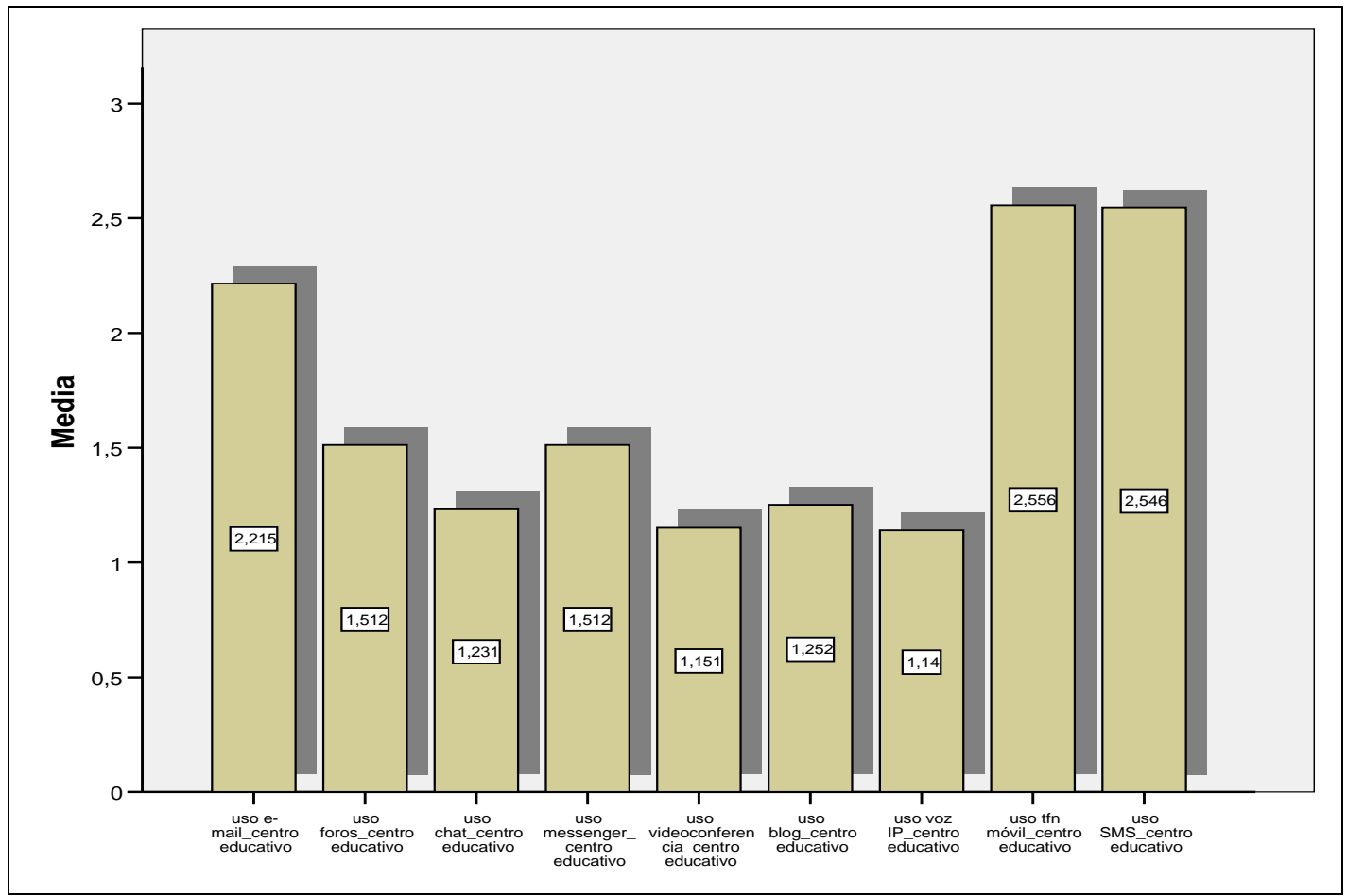

Gráfico 4. Escuela/Instituto/Facultad

\subsection{5.- En viajes/ desplazamientos}

En este contexto de movilidad sólo obtienen valoración de aprobado el móvil tanto para ser utilizado como medio de comunicación hablado como para mensajes.

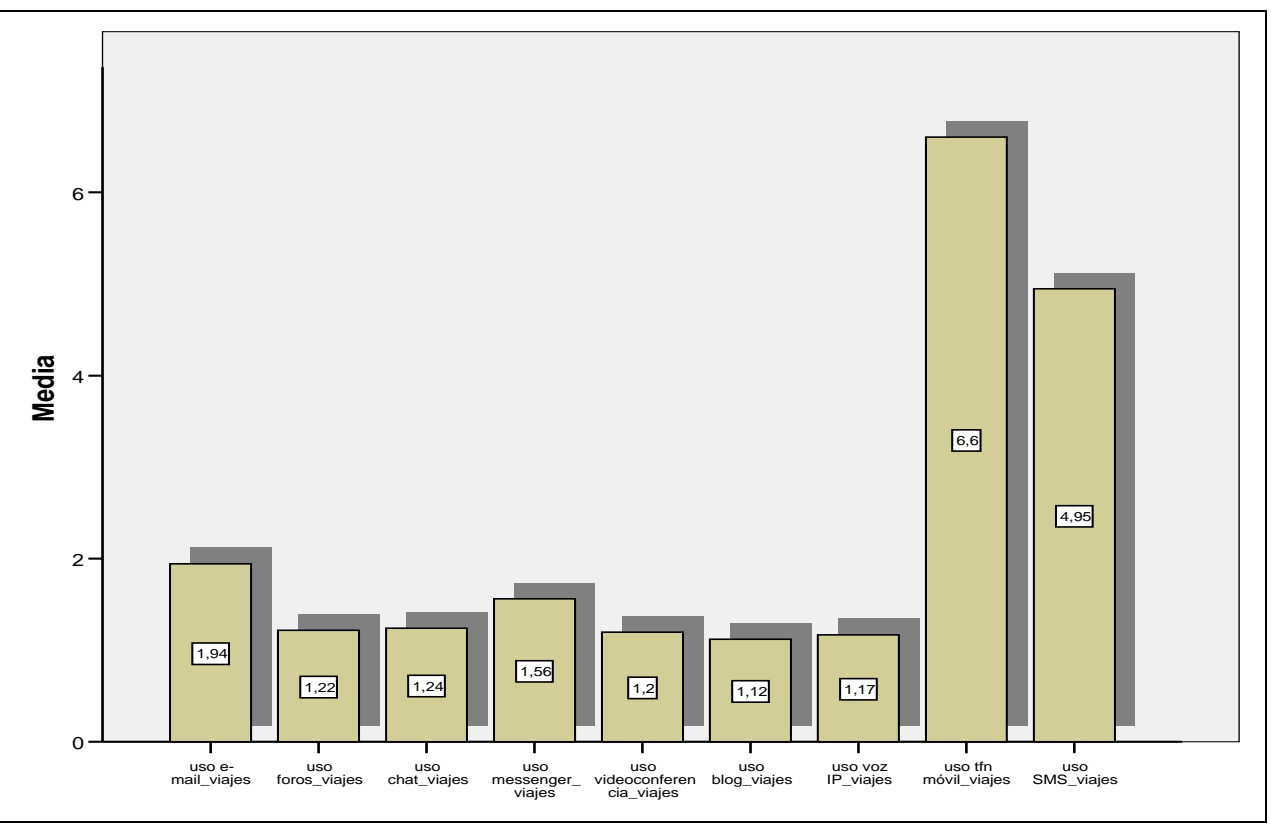

Gráfico 5. Viajes/desplazamientos

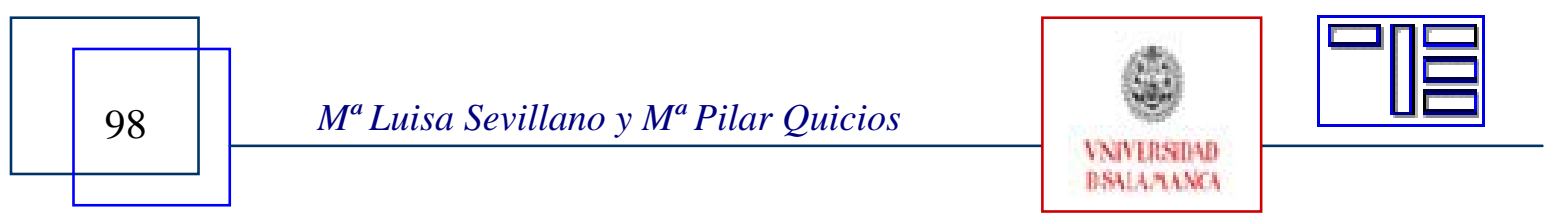




\subsection{6.- En cibercafé /acceso público Internet/bibliotecas}

Los resultados vuelven a coincidir con anteriores pero la valoración es baja (Gráfico 6). El comentario es complementario, dado que en algunos viajes, tanto el cibercafé, como las bibliotecas y los hoteles, que coinciden con la situación de viaje, han de ser objeto de un análisis más riguroso. Así, en la situación de viajes, es en los hoteles y cibercafés donde el viajero puede — con mayor tranquilidad acceder a los medios-.

En ambos casos, el uso hegemónico en los hoteles-viajes, es tanto el móvil y SMS, como el correo-electrónico y el Messenger. Las investigaciones evidencian que aunque con una distancia de más de 4 puntos, en los viajes y en el cibercafé, los medios más utilizados han sido: El teléfono móvil y el SMS.

Nuevamente, en el cibercafé el medio menos empleado es la videoconferencia (1.14), seguida del IP (1.17) y del blog (1.20) Estos valores reducidos contrastan con lo que ha de ser los cibercafés, las bibliotecas/intertecas del futuro, a las que han de asistir sus usuarios para emplear con satisfacción las TIC. La versatilidad del móvil, está permitiendo integrar la mayoría de los restantes medios, ¿pero será propiciadora de paz, serenidad y merecido ocio?

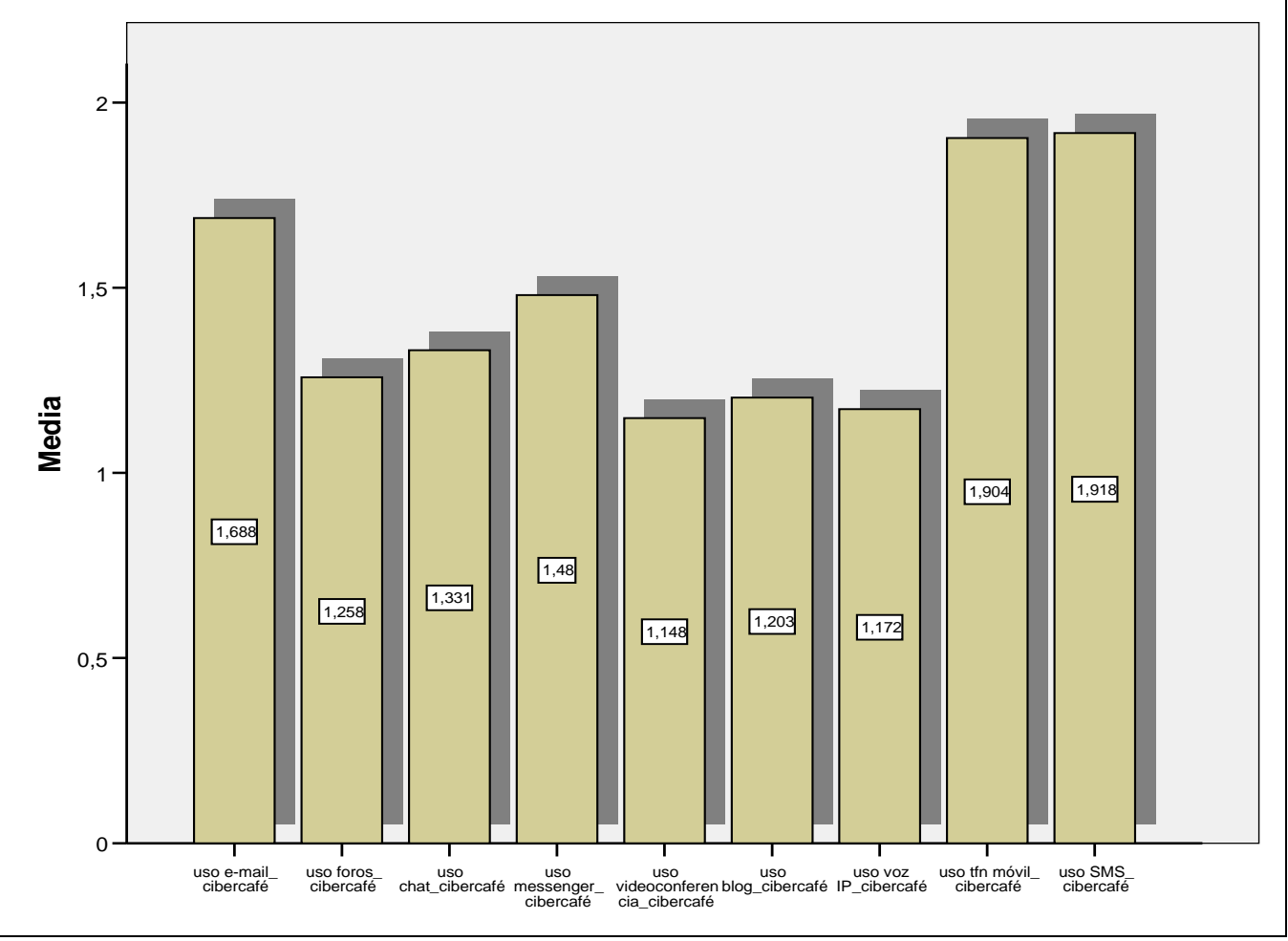

Gráfico 6. Cibercafé/bibliotecas

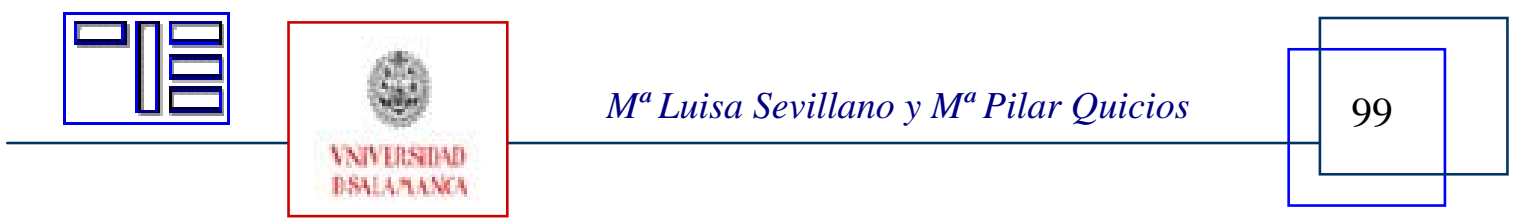


Revista Electrónica Teoría de la Educación.

Educación y Cultura en la Sociedad de la Información.

http://www.usal.es/teoriaeducacion

Vol.10. No2. Julio 2009

\section{3.- Los usos en función del contexto}

Mediante la aplicación del Análisis Factorial Confirmatorio, se aprecia como se puede constatar en las tablas siguientes, que los contextos hogar , trabajo, calle, centro educativo y viajes presentan una estructura bifactorial (Internet y telefonía). El primer factor se denomina Internet y está formado por las variables: e-mail, foros, Chat, Messenger, video, blog y vozip. El segundo factor denominado telefonía y agrupa las variables móvil y SMS.

Los análisis de los resultados obtenidos muestran que las herramientas virtuales estudiadas se utilizan fundamentalmente en los hogares y en el mundo del trabajo. Así, el correo electrónico alcanza en la casa una media ponderada de (4.95) frente al (3.85) que alcanza en el mundo laboral. Las conversaciones con telefonía móvil se utilizan en viajes (6.71) y en la calle (6.31) mientras que los SMS obtienen una mayor puntuación en el hogar (5.23) que en los viajes (5.10).

En definitiva, es el teléfono móvil en sus dos utilidades y el correo electrónico las herramientas más usadas tanto en el hogar como en la calle y los viajes. El resto de medios está infrautilizado. Dos pueden ser los motivos de su infrecuente uso, uno el desconocimiento de su funcionamiento y otro la falta de seguridad en la comunicación fuera del hogar, (E-mail (4.76), Foros (4.84), Chat (5.35), Messenger (4.81), Blog (4.85)). Otros escollos específicos a la hora de optar por celebrar una Videoconferencia (4.97) y usar Voz IP (4.91) es la movilidad. Las llamadas de teléfonos móviles (5.77) y mensajes de desde ellos (5.12) también tienen sus propios inconvenientes y éstos se sitúan en la carestía del servicio.

Claves para interpretar las tablas siguientes:

Se refieren a la frecuencia de uso de las diversas herramientas virtuales.

Columna 1= Contexto. 2= Ninguna. 3= Alguna vez. 4.= Muy ocasionalmente.

5.= Ocasionalmente. 6.= Según necesidades. 7.= Con cierta frecuencia. 8. $=$ Habitualmente. 9.= Muy habitualmente. 10. = Casi permanentemente. 11.= Permanentemente. 12. $=$ Media. 13. = Desviación típica

Tabla 12 Foros y listas de distribución

\begin{tabular}{|l|l|l|l|l|l|l|l|l|l|l|l|l|}
\hline 1. Contexto & 2 & 3 & 4 & 5 & 6 & 7 & 8 & 9 & 10 & 11 & 12 & 13 \\
\hline Hogar & $66,4 \%$ & $4,0 \%$ & $4,2 \%$ & $2,0 \%$ & $6,3 \%$ & $3,3 \%$ & $3,3 \%$ & $3,8 \%$ & $1,3 \%$ & $5,6 \%$ & 2,659 & 2,792 \\
\hline Trabajo & $82,7 \%$ & $3,9 \%$ & $2,6 \%$ & $0,9 \%$ & $2,0 \%$ & $1,6 \%$ & $1,3 \%$ & $1,9 \%$ & $1,1 \%$ & $2,0 \%$ & 1,761 & 2,031 \\
\hline Calle & $96,7 \%$ & $1,7 \%$ & $0,5 \%$ & & $0,3 \%$ & $0,1 \%$ & $0,2 \%$ & $0,1 \%$ & & $0,3 \%$ & 1,097 & 0,721 \\
\hline C.Educativo & $89,2 \%$ & $1,6 \%$ & $0,6 \%$ & $0,6 \%$ & $2,2 \%$ & $1,3 \%$ & $1,1 \%$ & $1,7 \%$ & $0,6 \%$ & $1,1 \%$ & 1,534 & 1,741 \\
\hline Viajes & $3,1 \%$ & $1,8 \%$ & $1,0 \%$ & $0,4 \%$ & $1,3 \%$ & $0,7 \%$ & $0,6 \%$ & $0,4 \%$ & $0,1 \%$ & $0,4 \%$ & 1,254 & 1,131 \\
\hline Cibercafé & $92,2 \%$ & $2,6 \%$ & $1,3 \%$ & $0,3 \%$ & $1,6 \%$ & $0,2 \%$ & $0,6 \%$ & $0,5 \%$ & $0,1 \%$ & $0,7 \%$ & 1,273 & 1,182 \\
\hline
\end{tabular}

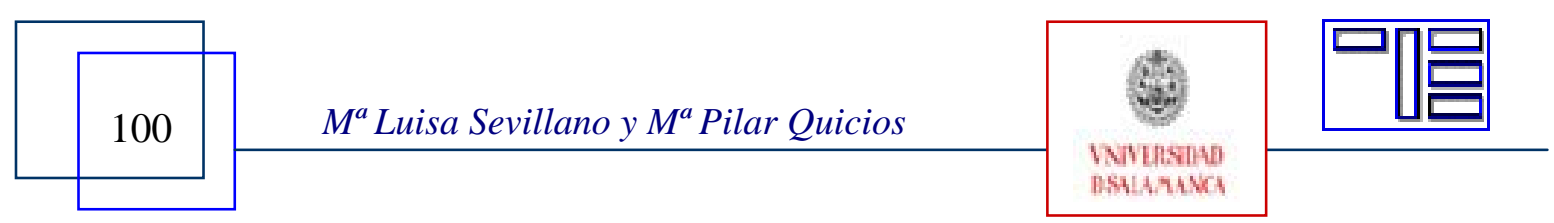




\begin{tabular}{|l|l|l|l|l|l|l|l|l|l|l|l|l|}
\hline 1. Contexto & 2 & 3 & 4 & 5 & 6 & 7 & 8 & 9 & 10 & 11 & 12 & 13 \\
\hline Hogar & $75,0 \%$ & $4,5 \%$ & $2,8 \%$ & $1,8 \%$ & $4,0 \%$ & $2,1 \%$ & $2,7 \%$ & $2,1 \%$ & $1,1 \%$ & $3,8 \%$ & 2,160 & 2,433 \\
\hline Trabajo & $93,4 \%$ & $2,0 \%$ & $1,1 \%$ & $0,9 \%$ & $0,4 \%$ & $0,6 \%$ & $0,8 \%$ & $0,4 \%$ & & $0,3 \%$ & 1,225 & 1,042 \\
\hline Calle & $97,5 \%$ & $0,2 \%$ & $1,0 \%$ & $0,2 \%$ & $0,2 \%$ & $0,5 \%$ & $0,2 \%$ & & & $0,1 \%$ & 1,085 & 0,615 \\
\hline C.Educativo & $93,7 \%$ & $1,5 \%$ & $0,8 \%$ & $0,5 \%$ & $0,9 \%$ & $0,7 \%$ & $0,6 \%$ & $0,3 \%$ & $0,3 \%$ & $0,7 \%$ & 1,265 & 1,225 \\
\hline Viajes & $94,0 \%$ & $0,4 \%$ & $1,1 \%$ & $0,4 \%$ & $1,0 \%$ & $0,9 \%$ & $0,7 \%$ & $0,6 \%$ & $0,3 \%$ & $0,4 \%$ & 1,273 & 1,226 \\
\hline Cibercafé & $91,3 \%$ & $1,6 \%$ & $1,4 \%$ & $1,3 \%$ & $1,4 \%$ & $0,8 \%$ & $0,6 \%$ & $0,6 \%$ & $0,5 \%$ & $0,8 \%$ & 1,357 & 1,376 \\
\hline
\end{tabular}

Tabla 14 Messenger

\begin{tabular}{|l|l|l|l|l|l|l|l|l|l|l|l|l|}
\hline 1. Contexto & 2 & 3 & 4 & 5 & 6 & 7 & 8 & 9 & 10 & 11 & 12 & 13 \\
\hline Hogar & $56,7 \%$ & $2,9 \%$ & $3,6 \%$ & $2,5 \%$ & $4,5 \%$ & $2,2 \%$ & $3,3 \%$ & $5,8 \%$ & $4,0 \%$ & $14,6 \%$ & 3,700 & 3,574 \\
\hline Trabajo & $86,5 \%$ & $2,5 \%$ & $2,3 \%$ & $1,1 \%$ & $1,9 \%$ & $1,5 \%$ & $1,2 \%$ & $0,6 \%$ & $0,4 \%$ & $1,9 \%$ & 1,573 & 1,758 \\
\hline Calle & $95,4 \%$ & $0,8 \%$ & $0,4 \%$ & $0,6 \%$ & $1,5 \%$ & $0,1 \%$ & $0,2 \%$ & $0,8 \%$ & $0,2 \%$ & & 1,184 & 0,957 \\
\hline C.Educativo & $88,1 \%$ & $1,9 \%$ & $1,5 \%$ & $0,9 \%$ & $2,9 \%$ & $0,7 \%$ & $1,4 \%$ & $0,9 \%$ & $0,5 \%$ & $1,3 \%$ & 1,522 & 1,667 \\
\hline Viajes & $88,1 \%$ & $1,1 \%$ & $1,1 \%$ & $1,3 \%$ & $1,8 \%$ & $0,8 \%$ & $2,1 \%$ & $1,6 \%$ & $0,2 \%$ & $1,8 \%$ & 1,601 & 1,851 \\
\hline Cibercafé & $87,8 \%$ & $2,6 \%$ & $1,6 \%$ & $1,0 \%$ & $2,5 \%$ & $0,9 \%$ & $0,8 \%$ & $0,8 \%$ & $0,7 \%$ & $1,4 \%$ & 1,511 & 1,657 \\
\hline
\end{tabular}

Tabla 15 Videoconferencia

\begin{tabular}{|l|l|l|l|l|l|l|l|l|l|l|l|l|}
\hline 1.Contexto & 2 & 3 & 4 & 5 & 6 & 7 & 8 & 9 & 10 & 11 & 12 & 13 \\
\hline Hogar & $81,0 \%$ & $3,2 \%$ & $2,6 \%$ & $1,2 \%$ & $3,9 \%$ & $1,8 \%$ & $1,7 \%$ & $2,0 \%$ & $0,6 \%$ & $1,9 \%$ & 1,835 & 2,050 \\
\hline Trabajo & $90,5 \%$ & $2,6 \%$ & $1,5 \%$ & $1,2 \%$ & $2,1 \%$ & $0,4 \%$ & $0,4 \%$ & $0,4 \%$ & $0,3 \%$ & $0,4 \%$ & 1,324 & 1,225 \\
\hline Calle & $96,4 \%$ & $0,9 \%$ & $0,7 \%$ & $0,5 \%$ & $0,5 \%$ & $0,2 \%$ & $0,2 \%$ & $0,2 \%$ & $0,2 \%$ & $0,2 \%$ & 1,133 & 0,842 \\
\hline C.Educativo & $95,2 \%$ & $1,3 \%$ & $0,8 \%$ & $0,5 \%$ & $1,0 \%$ & $0,2 \%$ & $0,2 \%$ & $0,3 \%$ & $0,1 \%$ & $0,3 \%$ & 1,174 & 0,941 \\
\hline Viajes & $94,5 \%$ & $0,7 \%$ & $1,2 \%$ & $0,6 \%$ & $0,5 \%$ & $0,8 \%$ & $0,8 \%$ & $0,2 \%$ & $0,2 \%$ & $0,6 \%$ & 1,239 & 1,149 \\
\hline Cibercafé & $94,5 \%$ & $1,3 \%$ & $1,5 \%$ & $0,6 \%$ & $0,8 \%$ & $0,5 \%$ & $0,2 \%$ & $0,3 \%$ & & $0,3 \%$ & 1,182 & 0,927 \\
\hline
\end{tabular}

Tabla 16 Blogs

\begin{tabular}{|l|l|l|l|l|l|l|l|l|l|l|l|l|}
\hline 1. Contexto & 2 & 3 & 4 & 5 & 6 & 7 & 8 & 9 & 10 & 11 & 12 & 13 \\
\hline Hogar & $80,1 \%$ & $2,9 \%$ & $3,1 \%$ & $2,4 \%$ & $2,9 \%$ & $1,5 \%$ & $2,1 \%$ & $1,6 \%$ & $0,8 \%$ & $2,6 \%$ & 1,887 & 2,134 \\
\hline Trabajo & $92,7 \%$ & $0,9 \%$ & $1,6 \%$ & $0,5 \%$ & $1,6 \%$ & $0,6 \%$ & $0,7 \%$ & $0,8 \%$ & $0,2 \%$ & $0,5 \%$ & 1,303 & 1,254 \\
\hline Calle & $97,7 \%$ & $0,7 \%$ & $0,2 \%$ & $0,1 \%$ & $0,3 \%$ & $0,3 \%$ & $0,3 \%$ & $0,2 \%$ & & & 1,082 & 0,628 \\
\hline C.Educativo & $93,5 \%$ & $1,0 \%$ & $1,0 \%$ & $0,7 \%$ & $1,3 \%$ & $0,8 \%$ & $0,9 \%$ & $0,2 \%$ & $0,1 \%$ & $0,3 \%$ & 1,256 & 1,122 \\
\hline Viajes & $96,2 \%$ & $1,1 \%$ & $0,7 \%$ & $0,5 \%$ & $0,3 \%$ & $0,1 \%$ & $0,3 \%$ & $0,2 \%$ & $0,1 \%$ & $0,3 \%$ & 1,135 & 0,855 \\
\hline
\end{tabular}

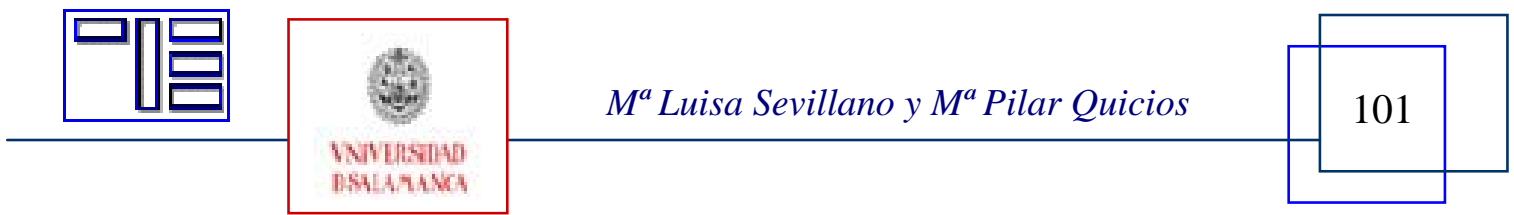


Revista Electrónica Teoría de la Educación.

Educación y Cultura en la Sociedad de la Información.

http://www.usal.es/teoriaeducacion

Vol.10. No2. Julio 2009

\begin{tabular}{|l|l|l|l|l|l|l|l|l|l|l|l|l|l|}
\hline Cibercafé & $94,7 \%$ & $1,4 \%$ & $0,7 \%$ & $0,3 \%$ & $1,0 \%$ & $0,2 \%$ & $0,3 \%$ & $0,2 \%$ & $0,5 \%$ & $0,6 \%$ & 1,215 & 1,114 \\
\hline
\end{tabular}

Tabla 17 Uso de la voz/imagen IP

\begin{tabular}{|l|l|l|l|l|l|l|l|l|l|l|l|l|}
\hline 1. Contexto & $\mathbf{2}$ & $\mathbf{3}$ & $\mathbf{4}$ & $\mathbf{5}$ & $\mathbf{6}$ & $\mathbf{7}$ & $\mathbf{8}$ & $\mathbf{9}$ & $\mathbf{1 0}$ & $\mathbf{1 1}$ & $\mathbf{1 2}$ & $\mathbf{1 3}$ \\
\hline Hogar & $81,9 \%$ & $3,3 \%$ & $2,5 \%$ & $1,8 \%$ & $2,6 \%$ & $1,7 \%$ & $2,6 \%$ & $0,8 \%$ & $0,8 \%$ & $2,0 \%$ & 1,777 & 1,985 \\
\hline Trabajo & $93,4 \%$ & $1,3 \%$ & $0,9 \%$ & $0,3 \%$ & $1,4 \%$ & $0,7 \%$ & $0,9 \%$ & $0,2 \%$ & $0,2 \%$ & $0,7 \%$ & 1,280 & 1,237 \\
\hline Calle & $96,1 \%$ & $0,7 \%$ & $0,8 \%$ & $0,2 \%$ & $0,7 \%$ & $0,5 \%$ & $0,2 \%$ & & $0,2 \%$ & $0,6 \%$ & 1,163 & 0,972 \\
\hline C.Educativo & $95,6 \%$ & $0,8 \%$ & $0,9 \%$ & $0,6 \%$ & $0,9 \%$ & $0,5 \%$ & $0,3 \%$ & $0,2 \%$ & & $0,1 \%$ & 1,152 & 0,821 \\
\hline Viajes & $95,5 \%$ & $0,6 \%$ & $0,6 \%$ & $0,5 \%$ & $1,0 \%$ & $0,2 \%$ & $0,5 \%$ & $0,3 \%$ & & $0,8 \%$ & 1,207 & 1,110 \\
\hline Cibercafé & $95,1 \%$ & $0,9 \%$ & $0,6 \%$ & $0,3 \%$ & $0,7 \%$ & $0,7 \%$ & $0,3 \%$ & $0,3 \%$ & $0,2 \%$ & $0,8 \%$ & 1,227 & 1,174 \\
\hline
\end{tabular}

Tabla 18 Teléfono móvil

\begin{tabular}{|l|l|l|l|l|l|l|l|l|l|l|l|l|}
\hline 1.Contexto & $\mathbf{2}$ & $\mathbf{3}$ & $\mathbf{4}$ & $\mathbf{5}$ & $\mathbf{6}$ & $\mathbf{7}$ & $\mathbf{8}$ & $\mathbf{9}$ & $\mathbf{1 0}$ & $\mathbf{1 1}$ & $\mathbf{1 2}$ & $\mathbf{1 3}$ \\
\hline Hogar & $11,1 \%$ & $5,9 \%$ & $8,1 \%$ & $5,6 \%$ & $13,7 \%$ & $7,7 \%$ & $10,0 \%$ & $11,8 \%$ & $7,0 \%$ & $19,1 \%$ & 6,024 & 3,013 \\
\hline Trabajo & $37,1 \%$ & $9,9 \%$ & $6,3 \%$ & $3,8 \%$ & $9,5 \%$ & $5,7 \%$ & $4,5 \%$ & $5,5 \%$ & $3,3 \%$ & $14,4 \%$ & 4,219 & 3,374 \\
\hline Calle & $12,4 \%$ & $4,6 \%$ & $5,2 \%$ & $3,9 \%$ & $12,1 \%$ & $9,2 \%$ & $9,7 \%$ & $12,7 \%$ & $8,6 \%$ & $21,6 \%$ & 6,312 & 3,055 \\
\hline C.Educativo & $67,9 \%$ & $4,1 \%$ & $3,9 \%$ & $2,7 \%$ & $5,2 \%$ & $3,5 \%$ & $2,8 \%$ & $2,6 \%$ & $1,2 \%$ & $6,0 \%$ & 2,573 & 2,758 \\
\hline Viajes & $14,7 \%$ & $2,9 \%$ & $3,8 \%$ & $3,9 \%$ & $6,9 \%$ & $6,1 \%$ & $9,1 \%$ & $13,4 \%$ & $10,8 \%$ & $28,5 \%$ & 6,715 & 3,218 \\
\hline Cibercafé & $81,8 \%$ & $3,3 \%$ & $1,9 \%$ & $0,9 \%$ & $2,4 \%$ & $1,4 \%$ & $1,5 \%$ & $1,5 \%$ & $0,8 \%$ & $4,5 \%$ & 1,926 & 2,343 \\
\hline
\end{tabular}

Tabla 19 SMS

\begin{tabular}{|l|l|l|l|l|l|l|l|l|l|l|l|l|}
\hline 1.Contexto & $\mathbf{2}$ & $\mathbf{3}$ & $\mathbf{4}$ & $\mathbf{5}$ & $\mathbf{6}$ & $\mathbf{7}$ & $\mathbf{8}$ & $\mathbf{9}$ & $\mathbf{1 0}$ & $\mathbf{1 1}$ & $\mathbf{1 2 .}$ & $\mathbf{1 3 .}$ \\
\hline Hogar & $22,6 \%$ & $9,5 \%$ & $6,1 \%$ & $3,6 \%$ & $12,5 \%$ & $6,7 \%$ & $6,8 \%$ & $8,3 \%$ & $6,3 \%$ & $17,5 \%$ & 5,232 & 3,347 \\
\hline Trabajo & $52,6 \%$ & $9,7 \%$ & $4,5 \%$ & $3,3 \%$ & $8,2 \%$ & $4,1 \%$ & $4,4 \%$ & $4,0 \%$ & $2,1 \%$ & $7,1 \%$ & 3,165 & 2,971 \\
\hline Calle & $29,4 \%$ & $7,4 \%$ & $5,9 \%$ & $2,9 \%$ & $11,0 \%$ & $4,7 \%$ & $8,9 \%$ & $8,0 \%$ & $5,8 \%$ & $16,1 \%$ & 4,956 & 3,421 \\
\hline C. educativo & $70,8 \%$ & $3,1 \%$ & $2,9 \%$ & $2,3 \%$ & $5,4 \%$ & $2,7 \%$ & $2,1 \%$ & $1,5 \%$ & $2,1 \%$ & $7,1 \%$ & 2,548 & 2,845 \\
\hline Viajes & $33,1 \%$ & $3,8 \%$ & $5,1 \%$ & $3,4 \%$ & $7,8 \%$ & $4,6 \%$ & $7,7 \%$ & $9,4 \%$ & $6,4 \%$ & $18,7 \%$ & 5,100 & 3,576 \\
\hline Cibercafé & $82,1 \%$ & $3,1 \%$ & $1,9 \%$ & $1,0 \%$ & $1,5 \%$ & $1,5 \%$ & $1,6 \%$ & $1,4 \%$ & $1,2 \%$ & $4,6 \%$ & 1,946 & 2,395 \\
\hline
\end{tabular}

\section{6.- DISCUSIÓN DE LOS RESULTADOS}

Los diversos análisis aplicados a los resultados obtenidos muestran que el hogar y el trabajo son los lugares privilegiados para el uso de las herramientas virtuales estudiadas. El correo electrónico alcanza una media ponderada en el hogar de 4. 95 y en el trabajo de 3. 85. Los foros/listas de distribución tienen valoración de 2, el Chat 2.16, la video-

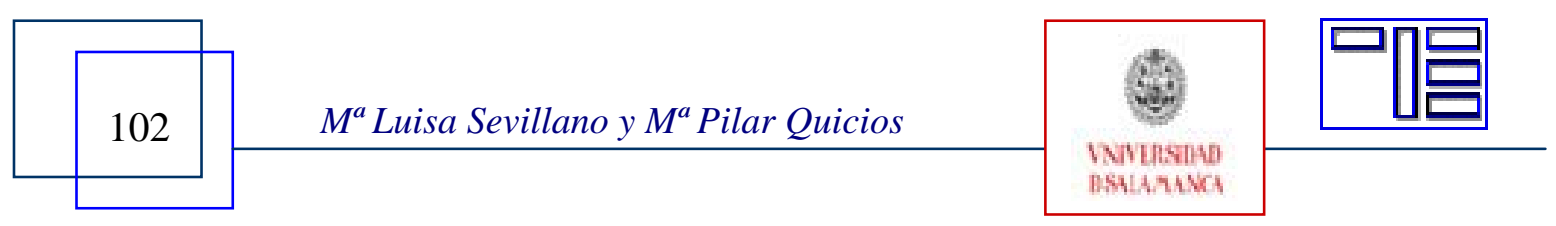




\begin{tabular}{|c|c|c|}
\hline 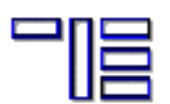 & $\begin{array}{l}\text { Revista Electrónic } \\
\text { Educación y Cultura en }\end{array}$ & $\begin{array}{l}\text { de la Educación. } \\
\text { dad de la Información. }\end{array}$ \\
\hline & http://www.usal.es/teoriaeducacion & Vol. 10. No2. Julio 2009 \\
\hline
\end{tabular}

conferencia 1.83, los blogs 1.89, Voz/ imagen IP 1.78, hablar por teléfono móvil se utiliza en viajes 6.71 y en la calle 6.31. Finalmente, mensajes por teléfono móvil/SMS tiene mayor valoración en el hogar (5.23) y en viajes (5.10).

Los tres medios que mayor valoración alcanzan y tienen mayor utilidad son: el teléfono móvil para hablar y para mandar mensajes y el correo electrónico. El lugar donde más se utilizan es en el hogar y en viajes/calle. El resto de medios está poco valorado quizás por desconocimiento de los mismos.

Una reflexión sobre causas de estos datos nos lleva a concluir que la seguridad, mejor dicho, la falta de seguridad en la comunicación (correo electrónico 4.76, foros 4.84, Chat 5.35, Messenger 4.81, blog 4.85) es el principal escollo para su bajo uso, fuera del hogar. Para la videoconferencia (4.97) y la voz IP (4.91) es la movilidad. Para el teléfono móvil voz (5.77) y teléfono móvil mensajes (5.12) es el coste del servicio.

\section{7.- CONCLUSIONES}

1. Herramientas más utilizadas La utilización de diversos medios en el entorno del hogar evidencia que dos medios han alcanzado un uso predominante, al obtener valores superiores a 5 (5.99 en el caso del teléfono móvil y 5.18 el SMS ). En los viajes estos valores llegan a una media de 6.6 y de 4.95 respectivamente. En la calle siguen siendo las herramientas más utilizadas con una ponderación media de 6.32 y 4.84. Y en centros de estudio como Escuela, Instituto o Universidad su uso es menor.

Las herramientas virtuales se han incorporado a la mayoría de los hogares consultados, pero el empleo de ellos, salvando los primeros citados y el uso del e-mail y del Messenger es reducido. A los hogares corresponde al empleo cultural de utilizar el teléfono móvil y los mensajes como el referente más habitual, entendiéndolo como espacio de comunicación adecuado, pero más centrado en las comunicaciones familiares, aunque la cultura del teletrabajo y del escenario plural de uso de algunos, está incorporando con frecuencia el correo electrónico y el Messenger, que integran la imagen y la sincronía del mensaje, que mejora la empatía entre los hablantes.

La reflexión en ambos ambientes, el hogar-familia y el escolar-comunidad educativa, es que en ambos se mantiene el conjunto de valores, pero el uso del móvil y del Messenger, ha de reducirse a escenarios de recreo y salas de docentes. Quizás esta realidad explique el descenso de uso.

2. Frecuencia de uso de las diversas herramientas En la categoría de más de cinco veces al día los resultados fueron: Páginas Web 17.6, Transferencia de archivos P2P 8.8, e-mail 19.6, foros 3.9, Chat 2.8, Messenger 8.5, Videoconferencia 1.3, Blog 1.8, Voz imagen IP 1.8.

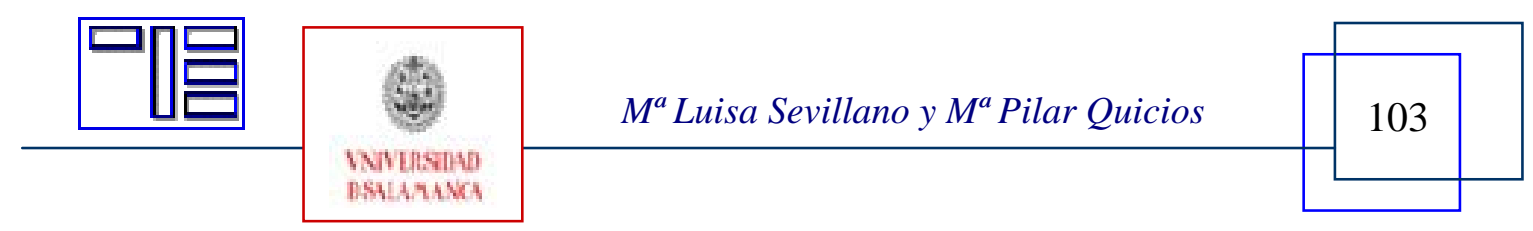


Constatamos en esta categoría una gran diferencia, focalizándose el uso frecuente en los correos y en las páginas web. En la categoría 1-4 veces al día las frecuencias registradas por herramienta fueron: Páginas web $28.0 \%$, e-mail $26.2 \%$, Transferencia de archivos $14.5 \%$, Messenger $13.3 \%$, foros $7.2 \%$, Chat $4.3 \%$, blog $2.9 \%$, Voz Imagen IP $2.7 \%$, videoconferencia $1.5 \%$. En la de 1 vez por semana encontramos que la páginas web registran $19.6 \%$ lo que nos habla de un segmento de población que va accediendo paulatinamente a este servicio, $17.4 \%$ para la técnica de transferencia de archivos, $20.3 \%$ e-mail, 14.4. \% foros, $7.9 \%$ chat, $16.0 \%$ Messenger, $5.5 \%$ videoconferencia, $5.6 \%$ blog y 5.7 \% para voz-imagen IP lo que nos indica el uso en crecimiento de la transferencia de datos y los foros.

3. Herramientas más utilizadas en el Trabajo El teléfono móvil y el correo electrónico emergen como medios más apropiados. No sorprende el reducido uso de los chat que ocupa la valoración más lejana, seguida por el uso IP, videoconferencia y blog, en una secuencia de baja estimación.

4. Teléfono móvil. Resulta ser el medio más utilizado y se apuntan como razones las siguientes virtualidades

Su funcionamiento: Del todo positivo 35. 0 \%, Positivo $21.0 \%$, Muy positivo $14.7 \%$, Bastante positivo $12.4 \%$.

La facilidad de usos: Del todo positivo 36.3 \%, Muy positivo 24.1, Positivo $19.4 \%$, Bastante positivo $9.9 \%$.

Su manejo similar a otras herramientas es apreciado como Positivo 24.7, Del todo positivo $18.2 \%$, Bastante positivo $16.5 \%$, Muy positivo $13.9 \%$.

Referente a las posibilidades que ofrece de utilizarse de modo combinado con otros medios los resultados fueron: Del todo positivo 22.5\%, Positivo 18.6 \%, Muy positivo 15.6 $\%$, Bastante positivo 14.6.

Acerca de si facilita su trabajo respondieron: Del todo positivo $32.2 \%$, Muy positivo $16.8 \%$, Positivo $11.9 \%$, Bastante positivo $9.6 \%$.

Si incrementa la posibilidades de ocio: Del todo positivo $30.5 \%$, Muy positivo $14.8 \%$, Positivo $12.6 \%$, Bastante positivo $10.7 \%$

5. El papel de los Cibercafés . Por su propia especificidad, sorprende que los valores alcanzados tanto en los usos del correo electrónico (1.688), en Messenger (1.48 ) y en blog (1.148), sean tan bajos. Estos valores reducidos contrastan con lo que han de ser los cibercafés, las bibliotecas/intertecas del futuro, a las que han de asistir sus usuarios para emplear con satisfacción y eficacia las TIC. La versatilidad del teléfono móvil, está permitiendo integrar los restantes medios. Así, el nuevo medio síntesis de computador y teléfono, en el que se integra la cámara digital, permitirá una forma más abierta, ligera e inmediata de potencialidad comunicativa.

6. Desventajas y dificultades de las diversas herramientas. Los Blogs y la Voz IP con una media de 4.75 y 4.71 respectivamente son las dos herramientas en las que la velocidad más incide para su valoración negativa en cuanto a uso. Las pocas posibilida-

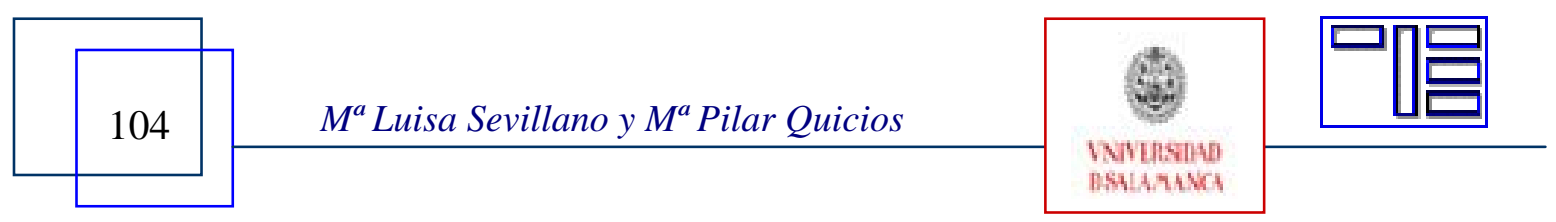




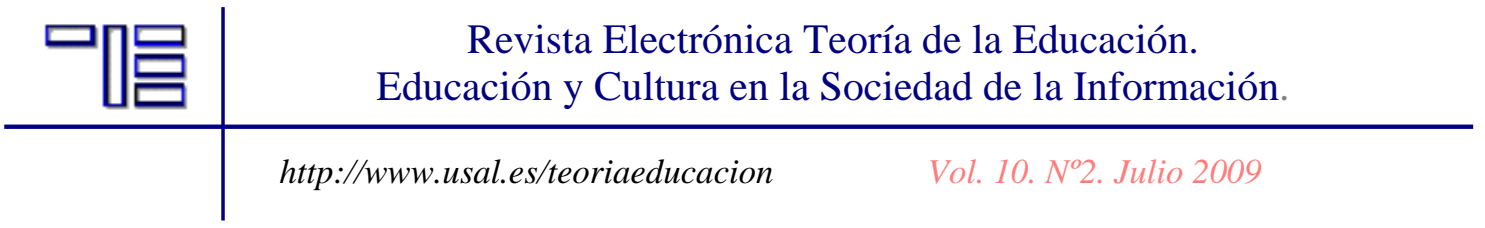

des de interactividad recibe altas puntuaciones de 4.66 y 4.46. En cuanto a la conectividad son la videoconferencia con una media de 4.88 y la Voz IP con media de 4.74 las que encuentran mayor dificultad.

Blogs, videoconferencia y Voz IP, son las tres herramientas que para su uso mayor dificultad presentan en cuanto a manejo. Al considerar la categoría eficacia son los blogs y la Voz IPE los peor valorados con medias respectivamente de 4.46 y 4.42. El coste de servicio alcanza negativamente, con valores de media de 5.77 y 5.12, al teléfono móvil y al SMS. La Voz IPE (media 4.91) y la videoconferencia son los dos medios que mayor dificultad encuentran en la variable movilidad.

En seguridad son el Chat media 5.35) y los blogs (media 4.85) los que mayor grado de dificultad presentan para su uso.

\section{8.- CATEGORIZACIÓN DE LAS VENTAJAS}

Analizados los textos sobre ventajas encontramos 80 aportaciones que se aglutinan en torno a las siguientes categorías positivas: Información, comunicación, rapidez, ruptura del concepto espacio, conocimiento, democratización, calidad de vida, consumo, formación permanente, dimensión profesional, colaboración, economía, ocio, interacción, actualización, calidad, creación, cultura, hipertextualidad, innovación, interconexión, libertad, mayor análisis, mentalidad joven, persistencia, relación, movilidad social, sociedad global, variedad.

\section{9.- CATEGORIZACIÓN DE DESVENTAJAS}

94 aportaciones que se pueden agrupar en 27 categorías, destacándose las siguientes : Abusos informativos (11.94 \%), tanto en el uso como en la divulgación de la información, saturación informativa (11.94 \%) manifestada en el exceso de información, los monopolios (10.45 \%) que influyen sobre las personas, la desigualdad (8.96 \%), la falta de fiabilidad (7.46 \%) generando inseguridad en las fuentes consultadas, las ilegalidades (7.46 \%) que se manifiestan en las actividades ilícitas y en los abusos en las transaciones económicas.

También son de importancia aunque en menor grado los cambios rápidos mal aplicados que desconciertan, el analfabetismo patente en el desfase de importantes colectivos, el consumismo por exceso y sin sentido, la desigualdad en los países, el desorden material, la pasividad, el aislamiento que provoca en las personas, la inseguridad, el cambio de relaciones, el exceso de tiempo que requiere su dominio, la pérdida de privacidad, los condicionantes y la resistencia que generan a determinadas edades.

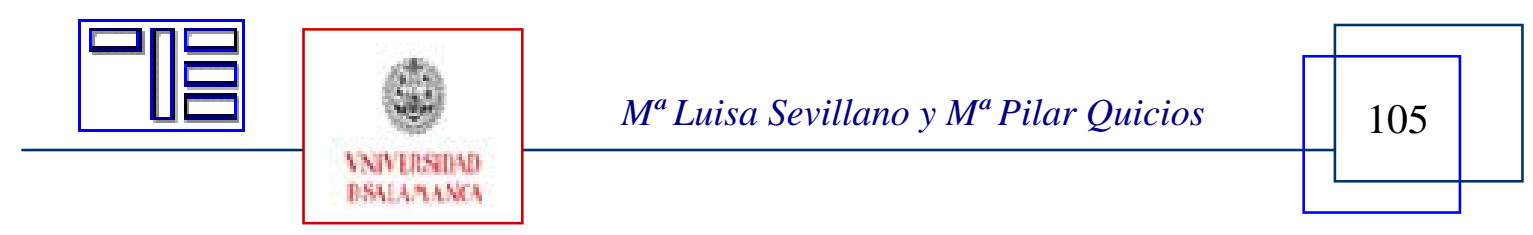


Revista Electrónica Teoría de la Educación.

Educación y Cultura en la Sociedad de la Información.

http://www.usal.es/teoriaeducacion

Vol.10. N². Julio 2009

\section{0.- LIMITACIONES DEL ESTUDIO}

Los propios conceptos nucleares de esta investigación como son las herramientas virtuales y la formación a lo largo de la vida, se encuentran en fase de desarrollo, delimitación y crecimiento. Dada igualmente la gran diversidad y desigualdad existentes en cuanto a posibilidades de acceso seguro a las redes, parece ilusorio pensar en una amplitud de contexto para su uso frecuente.

Sigue habiendo una gran brecha digital y unas carencias de oportunidades en el disfrute de estas nuevas herramientas. Se constata una falta de alfabetización conceptual y práctica que ha sido mal sustituida por un deseo de rápido acceso a todo y especialmente a lo más novedoso y moderno. Falta la serenidad, la reflexión los diagnósticos de ventajas, inconvenientes, tiempos a emplear, manuales de ayuda a conocer y aplicar, potencialidades de cada una de las herramientas y sus usos más adecuados.

\section{1.- PROSPECTIVA}

En los viajes se evidencia que son el uso de blog (1.2), seguido del empleo de IP (1.17) y de las videoconferencia (1.2) las herramientas virtuales que ocupan los valores más bajos; coincidentes con los obtenidos en el cibercafé. Las virtualidades de estas tecnologías son intensas y la posibilidad de intercambiar información directa y empática es posible. Esta realidad nos lleva a plantearnos su virtualidad futura, o su potencialidad informativa y de usuabilidad a medio plazo.

Los hogares son escenarios de vida que incorporan sucesivamente nuevos medios y entre ellos, los verdaderamente frecuentes corresponden al empleo cultural de utilizar el teléfono móvil y los mensajes como el referente más habitual, situando el hogar como un espacio de comunicación adecuado, pero más centrado en las comunicaciones familiares, aunque la cultura del teletrabajo y del escenario plural de uso de algunos, está incorporando con gran frecuencia el correo electrónico y el Messenger, que integran la imagen y la sincronía del mensaje, que mejore la empatía entre los hablantes.

La utilización más reducida de la videoconferencia y del IP, se puede deber a la mayor exigencia tecnológica de ambos y al escaso uso cultural de los mismos en los hogares. No obstante la reducción de costes, la diáspora cada vez mayor de familiares y amigos, las necesidades de comunicaciones familiares puede hacer de ellos herramientas a integrar con más frecuencia también en el ámbito familiar. Análoga reflexión es válida para ambientes profesionales ante la creciente diversificación de los mercados.

La llegada de la formación a los microámbitos rurales en cuestiones de ocio, salud, consumo, ciudadanía, alimentación, actividades culturales, etc. permite auspiciar igualmente una mayor presencia de las herramientas virtuales, a medida que la banda ancha se ruralice.

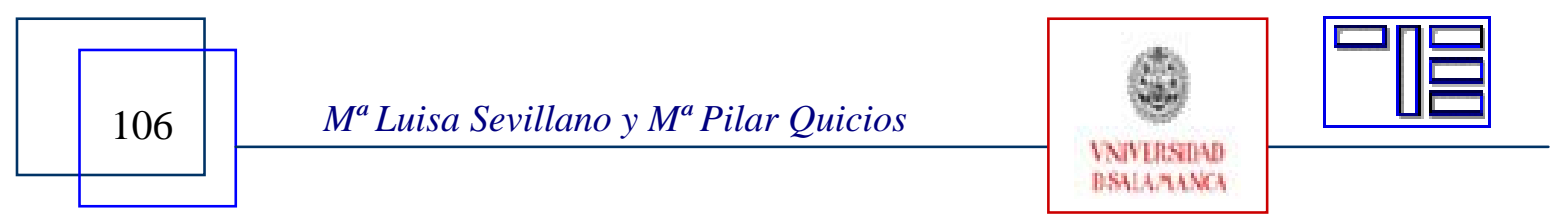




\section{REFERENCIAS BIBLIOGRÁFICAS}

CHAN M. E. (2005): Competencias mediacionales para la educación en línea. Revista Electrónica de Investigación Educativa, 7 (2). 1-21 Consultado el día 21 de Diciembre de 2008 en: http://redie.uabc.mx/vol7no2/contenido-chan.html

COLLIS, B y MOONEN, C. (2007): Flexible learning in a digital world. Open and distance learning series. London: Kegan Page Ltd

COM (2005): E-learning. Concebir la educación del futuro. Decisión del Parlamento Europeo y del Consejo

GERHARD, M. y KLINGER, W. (2006): Medienerziehung in der Zukunft. Media Perspektive. (2) pp. 75-90

GROS, B y MARIELLA, A. (2004): Estudio sobre el uso de los foros virtuales para favorecer las enseñanzas colaborativas en la enseñanza superior En Teoría de la Educación. N. 5, 1-13. Consultado el 21 de Diciembre de 2008

http//.www3.usal.es/ teoriaeducacion/rev_numero_05/n5_art_gros_adrian.htm - 40k -

LIEGLE, J. and MESO, P. (2007): Evaluation of a Virtual Lab Environment for Teaching Web Application Development. Informations Systems Education Journal. Vol. 5 (7). 1-11

ONRUBIA, J. (2005): Aprender y enseñar en entornos virtuales: actividad conjunta, ayuda pedagógica y construcción del conocimiento. RED: Revista de Educación a Distancia, 1-17. Consultada 21 de Diciembre 2008. http://www.um.es/ead/red/M2/

RÜDIGER, F. (2005): Kritische Medienkompetenz als Unterscheidungs- und Rahmungskompetenz. En Kleber, H. Perspektiven der Medienpädagogik in Wissenschaft und Praxis. München. KoepadVerlag

SEVILLANO, M. L. (2006): Competencias y Conocimientos sobre Herramientas virtuales. Formato de archivo: PDF/Adobe Acrobat-Versión en HTML. http://www.formatex.org/micte2006/pdf/27-31.pdf- Enseñanza: anuario interuniversitario de didáctica (23), 2005, pp. 387-402. ISSN 0212-53747

TULODZIEKI, G. (1999): Kompetenzen die studierende der Lehrämter während der universitären Ausbildung erwerben sollten. En Theuner H. Medienkompetenz. Munich Koepadverlag

Para citar la presente editorial puede utilizar la siguiente referencia:

SEVILLANO, M.L. \& QUICIOS, M. P. (2009): "La influencia del contexto en los usos de las herramientas virtuales”. En ORTEGA SÁNCHEZ, I. FERRÁS SEXTO, C. (Coord.) Alfabetización Tecnológica y desarrollo regional [monográfico en línea]. Revista Electrónica Teoría de la Educación: Educación y Cultura en la Sociedad de la Información. Vol. 10, $\mathrm{n}^{\circ}$ 2. Universidad de Salamanca [Fecha de consulta: $\mathrm{dd} / \mathrm{mm} /$ aaaa $]$.

http://www.usal.es/ teoriaeducacion/rev_numero_10_02/n10_02_sevillano_quicios.pdf ISSN: 1138-9737

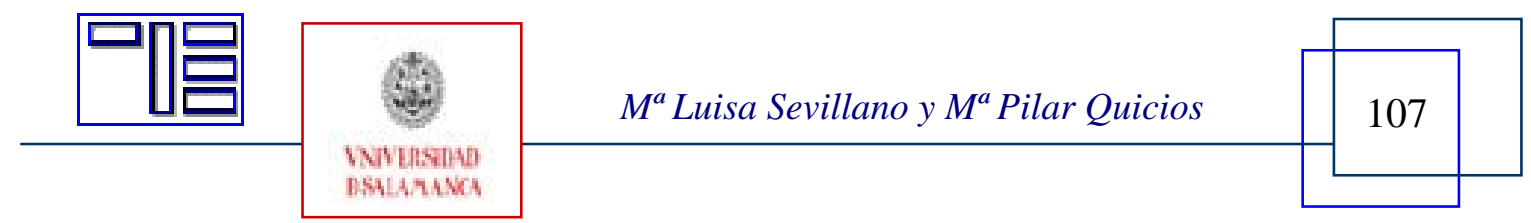

